\title{
The essential roles of chemistry in high-throughput screening triage
}

It is increasingly clear that academic high-throughput screening (HTS) and virtual HTS triage suffers from a lack of scientists trained in the art and science of early drug discovery chemistry. Many recent publications report the discovery of compounds by screening that are most likely artifacts or promiscuous bioactive compounds, and these results are not placed into the context of previous studies. For HTS to be most successful, it is our contention that there must exist an early partnership between biologists and medicinal chemists. Their combined skill sets are necessary to design robust assays and efficient workflows that will weed out assay artifacts, false positives, promiscuous bioactive compounds and intractable screening hits, efforts that ultimately give projects a better chance at identifying truly useful chemical matter. Expertise in medicinal chemistry, cheminformatics and purification sciences (analytical chemistry) can enhance the post-HTS triage process by quickly removing these problematic chemotypes from consideration, while simultaneously prioritizing the more promising chemical matter for follow-up testing. It is only when biologists and chemists collaborate effectively that HTS can manifest its full promise.

\section{HTS \& medicinal chemistry as partners in lead discovery}

An increasing number of screening centers are influencing the discovery of potential new therapeutics. These screening centers are being established in the academic environment, modeling themselves on their industrial counterparts [1-5]. However, this can result in unexpected challenges when it comes to later stages in the therapeutic discovery process. To ensure that high-throughput screening (HTS) output will be as fruitful as possible requires the collaboration and (often) early intervention of medicinal chemists trained in the art and science of HTS triage. Typically, industry has centralized these early stages of drug discovery into groups like the Lead Discovery Group at the former Pfizer site in Ann Arbor (MI, USA). That group consisted of approximately 30 experts in medicinal chemistry, cheminformatics, and analytical chemistry and purification sciences. One of the strategies behind having a single group perform unified HTS triage was to allow for rapid knowledge sharing. The same, or similar, library was constantly being interrogated by a wide variety of targets, and the recognition of actives and hits across these targets was continually bringing potentially nonselective and other so-called 'bad-actor' compounds to light. Grouping experts together led to a more facile sharing of this information and other, making the art of HTS triage more efficient. To our knowledge, all major pharmaceutical companies engaged in HTS have some sort of dedicated triage operation, large investments which should testify to the essential nature of this discipline in early drug discovery.

'Triage', as commonly understood, applies to the classification of human trauma patients into those who are likely to live, those who are likely to die, and those for whom immediate care might make a significant difference in their health outcome. This system may have originated on the battlefields of the first world war $[6,7]$. The ability to triage patients is a combination of art and science, some-
Jayme L Dahlin 1,2

\& Michael A Walters*,3

'Department of Molecular Pharmacology

\& Experimental Therapeutics, Mayo

Clinic College of Medicine, Rochester, MN 55905, USA

${ }^{2}$ Medical Scientist Training Program, Mayo Clinic College of Medicine, Rochester, MN 55905, USA

${ }^{3}$ Institute for Therapeutics Discovery \& Development, University of Minnesota, Minneapolis, MN 55414, USA *Author for correspondence: mwalters@umn.edu 
thing that is learned with extensive field experience. In a somewhat analogous process (although, admittedly, the outcomes are far less significant in human terms), HTS triage involves the classification, or prioritization, of hits from screening campaigns into compounds that are likely to survive further investigation, those that probably have no chance of succeeding either as probes (tool compounds), and those that fall into an intermediate classification where intervention could make a significant difference in their survival. Like its hospital and battlefield counterpart, HTS triage is also a combination of science and art, a process that is learned from extensive laboratory experience. Not every active can be saved, some cases will always be recognized as hopeless, while a third group will have structures and calculated or measured physicochemical properties where even seasoned experts are hard pressed to guess the potential outcomes offered by extensive screening and medicinal chemistry follow-up. Triage always has as its bottom line limited resources, and a major task of those performing HTS triage is directing the 'best' use of these finite resources [8]. With unlimited resources, all HTS actives could be carried on through increasingly expensive active-to-hit or lead assays and synthetic chemistry. However, unlimited resources are rarely the case in the pharmaceutical industry and even rarer in the academy. That being said, even medicinal chemistry experts are not in agreement over what constitutes a 'beautiful' compound or what will ultimately progress to a bona fide lead [9]. Therefore, the more potential resources are limited, the more sense it may make to err on the side of caution and apply higher bars to continuation of work on hits. Of course, the nature of the target (e.g., value, novelty and difficulty) plays an important role in this triage, sometimes lowering the bar to the follow-up of active compounds.

In our opinion some of the key places in the process of 'library-to-lead' where expertise in HTS triage can make a significant impact on the success of a drug discovery project are shown in Figure 1. Note that these contributions can begin well before an HTS is completed, and well after the 'cherry-picking' of screening hits, meaning the triage process has essential roles throughout the early discovery process. We should note that the definition of success may be a rapid acknowledgement by the project team that the search for chemical matter was unsuccessful, thereby preventing the consumption of resources on a project with little or no odds of succeeding. In the same fashion that the aforementioned 'Lead Discovery Group' was composed of experts in multiple fields, we believe the most effective HTS triage takes place in the hands of scientists with solid expertise in medicinal chemistry (with its grounding in synthetic chemistry and biological processes), cheminformatics and purification sciences [10]. This partnership of chemistry with the biological and biophysical assay methods of HTS offers the best chances of probe and drug discovery [11]. In this article, we highlight the importance of this partnership in HTS triage and lead discovery, and provide vignettes of our experiences in this area.

\section{The importance of library design in HTS triage: you will only find what you screen}

The goal of all screening methods is to find active compounds that can provide the starting points for compound optimization with respect to target binding, and physicochemical and toxicological properties. Of these, binding and physicochemical properties are often of primary concern in the early stages of drug discovery, given that toxicological challenges can often not typically be predicted accurately a priori and will most likely need to be remediated in the optimization process [12-14]. That being the case, the more the compounds that populate the screening library match the desired parameters in each of these attributes (e.g., drug/lead-like, appropriate physicochemical properties), the shorter, typically, will be the optimization of the compound to preclinical candidate status. Therefore, the principles of medicinal chemistry should be considered in assembling a high-quality chemical library within the context of what is commercially available or imaginable.

Two methods of discovering active compounds are commonly employed: high-throughput and fragmentbased screening. A third method of finding actives, virtual HTS (vHTS), produces recommendations of subsets of compounds to test in HTS or lower-throughput assays. The rough spectrum of libraries available to researchers is shown in Figure 2, with some examples of specific libraries that have been found to be useful. The GDB-13 is a computationally enumerated and curated collection of small organic molecules containing up to 13 atoms of $\mathrm{C}, \mathrm{N}, \mathrm{O}, \mathrm{S}$ and $\mathrm{Cl}$ or less $(\sim 977 \mathrm{M})$ and ZINC is combination of several commercial libraries $(35 \mathrm{M})[15,16]$. The CAS (CAS Registry) library (81 M substances) is shown here as bridging 'virtual' and 'tangible' designations because many of the compounds in that collection are commercially available [17]. The CAS library is not currently available for vHTS, but is extraordinarily helpful in sifting through potential actives with respect to their 'natural histories' (vide infra). eMolecules ( $6 \mathrm{M})$ contains compounds that are largely commercially available and are curated on a routine basis [18]. The GPHR (gopher) library is our in-house screening set of approximately $0.25 \mathrm{M}$ compounds, close to the size and chemical composition of many typical academic libraries [Dahlin JL, WaLters MA, Unpublished Data]. 


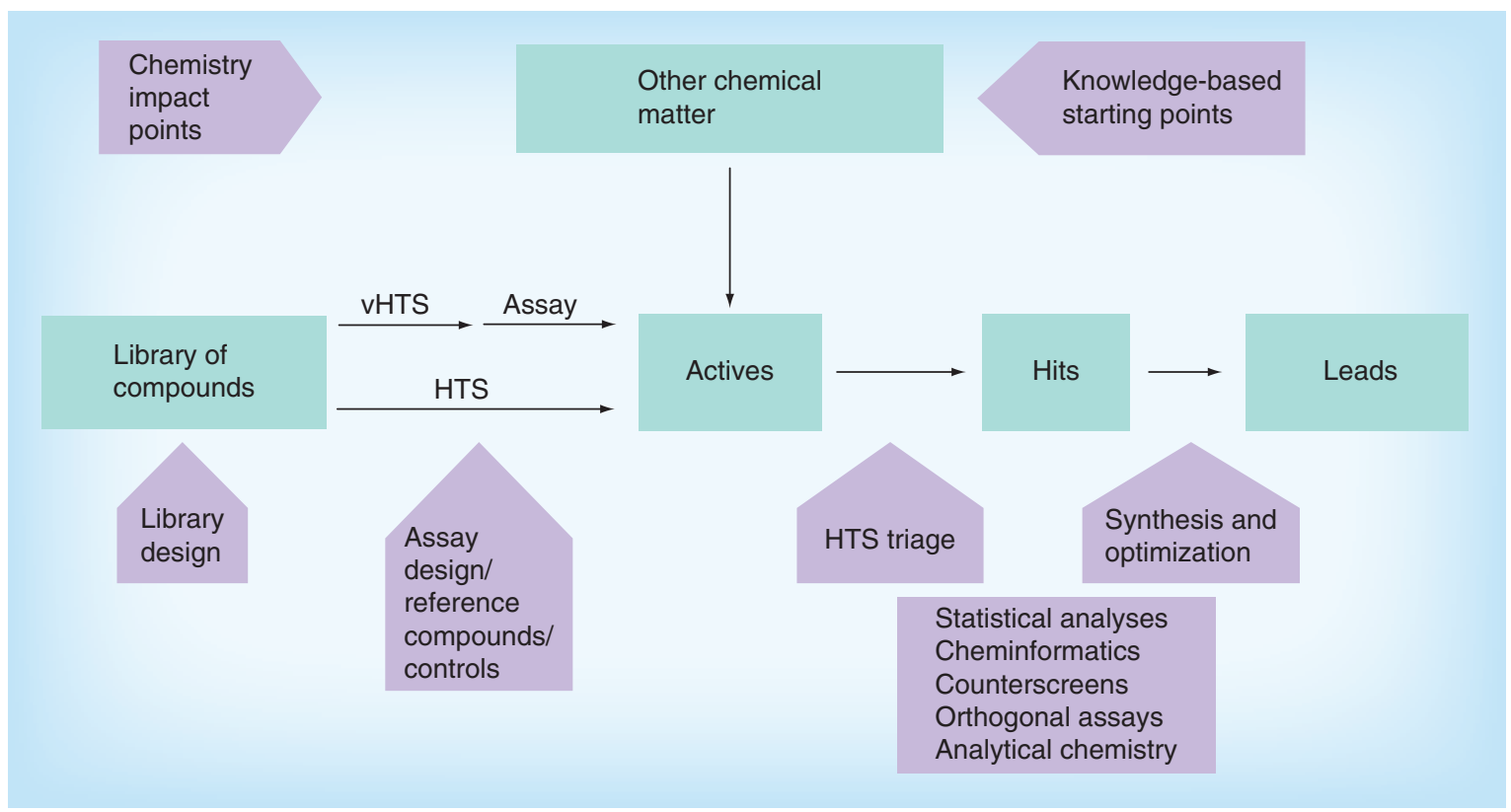

Figure 1. Primary impact points for chemistry in high-throughput screening and early lead discovery. The location of impact points covering the pre- and post-HTS stages highlights the importance of forming early partnerships between chemists and biologists in order to increase the chances of project success.

HTS: High-throughput screening; vHTS: Virtual high-throughput screening.

HTS is based on the premise that active compounds (e.g., $\left.\mathrm{IC}_{50}<25 \mu \mathrm{M}\right)$ can be discovered by interrogating targets with a large number of real, diverse and quality substances. The term 'real' here means compounds that are physically present in a screening collection. Other gross library descriptors can be used. 'Tangible' refers to compounds that are either commercially available or known to be amenable to facile preparation. 'Virtual' refers to a spectrum of compounds that can be easily prepared (overlaps with tangible), have been prepared, or might not even be capable of being prepared. This premise raises the questions of how large a library is large enough, how diverse is diverse enough, and what quality is good enough? Typical industrial screening libraries of small molecules contain on the order of 1-5 M compounds, while most academic screening libraries are on the order of $0.5 \mathrm{M}$ compounds [19]. At one point it was hypothesized that a library of $<25-50 \mathrm{~K}$ compounds '("Holy Grail” Library) would be sufficient to find weak actives against any target [20]. At the other extreme, a library of up to $10 \mathrm{M}$ compounds was proposed during the 'combinatorial era' by at least one major pharmaceutical company. Neither of these extremes worked in practice and the current thinking is that all of the commercially available compound space ( 6-8 M compounds) can be adequately covered by a library of around the size of most academic or non-forprofit screening groups [21]. Real compound library size is dictated by cost, storage and equipment throughput, among other considerations. Chemical diversity is best insured by having several representatives of each compound scaffold in the library to help validate actives (validated hits are more likely to arise when several compounds containing the same scaffold are found in the initial HTS). Quality can be judged by a number of filters such as rapid elimination of swill (REOS), Pan-assay interference compounds (PAINS), physicochemical properties, number of $\mathrm{sp}^{3}$ atoms, $\mathrm{RO} 5$ and $\mathrm{RO} 3$, among others [22-24]. It has been documented that compound beauty (quality) is truly in the eyes of the beholder. Moreover, these studies typically have used as the beholders several expert medicinal chemists who have had extensive experience in compound optimization and drug discovery and development [25-27]. Therefore, if the number and expertise of those reviewing the libraries that constitute an HTS collection (or screening output) are necessarily limited in expertise (e.g., as at an academic screening center), the use of standard, industrial-strength compound filters allows the collective wisdom of many medicinal chemistry experts to be virtually and effectively brought to the table.

\section{Key term}

PAINS: Pan-assay interference compounds; a series of compounds identified by Baell et al. that showed bioactivity in multiple AlphaScreen HTS campaigns, irrespective of the biological target. Compounds containing the PAINS substructures should generally be considered unsuitable as leads for chemical probes or medicinal chemistry optimization. The chemical mechanism(s) behind their promiscuous bioactivities vary depending on chemotype. 


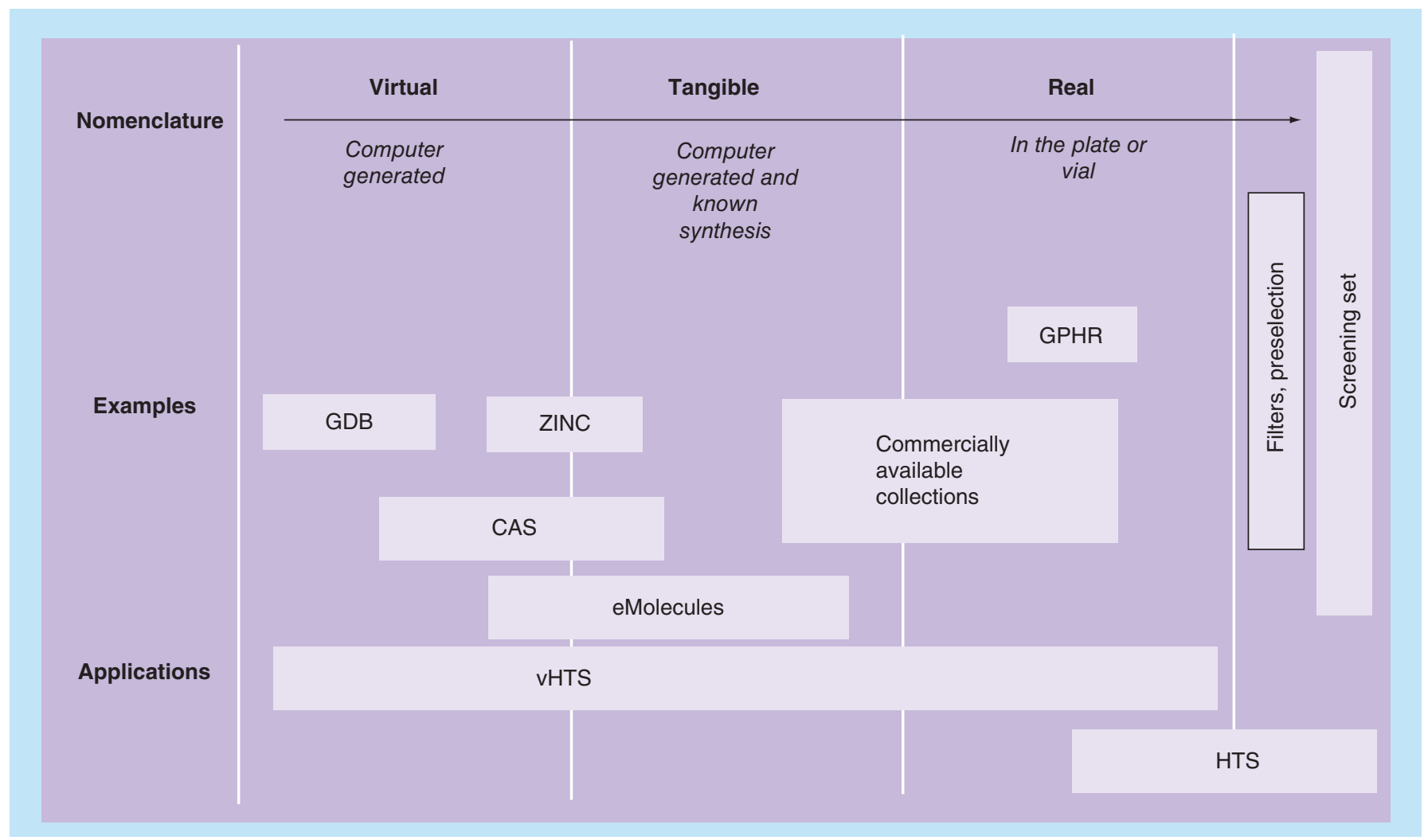

Figure 2. Libraries, their uses and the development of a quality screening set. Placement of representative libraries in this space is necessarily subject to debate. For example, virtual libraries of real compounds can be used in vHTS, and the GDB contains commercially available compounds.

HTS: High-throughput screening; vHTS: Virtual high-throughput screening.

A comparison of size and quality parameters for a few typical libraries is shown in Table 1. It is important to note that even the most carefully tended screening libraries contain approximately 5\% PAINS, although this is not appreciably more than what exists in the universe of commercially available compounds (eMolecules). This must be kept in mind when performing 'active' triage. It is probably worth rigorously removing these types of interference compounds from libraries designed for virtual screening campaigns [28]. Academic libraries tend to be composed primarily of vendor-supplied compounds (although some institutions have developed proprietary libraries) while industrial libraries are largely compounds that are proprietary to the company. These so-called 'legacy' compounds have been prepared during project-related medicinal chemistry campaigns or specifically in file enrichment endeavors (Box 1). Legacy compounds naturally have an understanding of medicinal chemistry principles 'baked' into them; we suspect that this is not typically the case with many vendor-supplied compounds.

Fragment-based screening (FBS) has been successfully applied to find active compounds for many years $[34,35]$. In this process, libraries of compounds with molecular weights less than approximately 250 and con- taining on the order of 5-10 K compounds are assayed at high concentrations in order to detect weak binding. Small fragments can sample a chemical space the size of a much larger library, and there are several commercially available fragment libraries. Three important benefits of FBS are: libraries are much smaller, thereby requiring fewer technical resources; since the compounds must be soluble to be screened at high concentrations, good physicochemical properties are typically already present in the actives; and the method of detection (e.g., XRD and SPR, among others) typically insures target engagement. In this case, fragment optimization to full hit compound typically consists of fragment growing, linking or merging. These processes are heavily dependent on medicinal chemistry. The major challenges in FBS are the specialized equipment involved, the (relatively) higher compound concentrations used for screening and their potential for nonspecific target engagement, and the potentially challenging optimization processes, but many examples of success in this area have been reported [36,37]. The same fundamental principles used in conventional HTS triages are applicable to FBS, such as the elimination of problematic chemotypes, although the continued growth of fragment-based methods and the 
Table 1. Four representative chemical libraries.

\begin{tabular}{|c|c|c|c|c|c|}
\hline Collection & Compounds (n) & HTS & REOS & PAINS & PAINS (\%) \\
\hline MLSMR & 314,651 & 7160 & 79,695 & 18,654 & 6 \\
\hline Academic A & 208,887 & 5082 & 59,041 & 10,098 & 5 \\
\hline Academic B & 234,304 & 3287 & 41,134 & 9831 & 4 \\
\hline eMolecules & $6,580,176$ & 200,805 & NC & 366,939 & 6 \\
\hline
\end{tabular}

expansion of knowledge will undoubtedly shed light on triage-related nuances specific to FBS.

vHTS can greatly expand the universe of compounds for screening since it allows the exploration of real, tangible and virtual space. As contrasted with the screening of real compounds, the major bottleneck in virtual screening is dictated by throughput of the computational method. The more computationally demanding the method, the smaller the number of compounds that can be screened in a reasonable amount of time. The typical workflow for virtual screening, therefore, involves development of a model for the target (or ligand), creation of the chemical space of choice by filtering (target or ligand based) of large virtual libraries, the actual computations (simulations), and then computational prioritization of compounds to enter into the 'real' assay. vHTS can effectively enrich the output of HTS by focusing that process on the compounds that are most likely to engage the target. Several examples of this type of enrichment exist [38]. However, many caveats apply to virtual screening [39], and here, as in HTS, the compounds that emerge are only as good as the skills of the scientists that choose to screen, test and triage them. While many practitioners of vHTS are also medicinal chemists, we feel it is imperative for experts in HTS triage be involved in evaluating the libraries to be used in the vHTS process (including the selection of compounds cherry-picked to be tested experimentally) and that vHTS be heavily influenced by the application of known quality filters prior to screening. Eventually compounds that arise from vHTS will need to be confirmed experimentally and possibly optimized at later stages, and the better these compounds adhere to the established norms for compound quality, the more efficient the optimization process may be.

\section{Other approaches to chemical matter}

Chemical matter can be defined as the collection of compounds that will be assayed, at any level of throughput, for a given biological target. While lead discovery is often predicated on HTS, other starting points for chemical matter should always be considered when exploring a new or previously screened target (Figure 3). This multifacetted approach is the hallmark of true lead discovery and is typically performed in parallel with HTS. These methods can be considered to be complementary to HTS, but are knowledge- rather than 'haystack'-based. Diverse approaches to chemical matter have been reviewed, vHTS being one of them [40]. Other approaches involve the use of known compounds from the literature (both journals and patents) that act at the target, other reported screens for the same target, and the reuse of preclinical pharmaceutical or biotechnology company compounds (Box 2).

\section{The critical roles of medicinal chemistry in successful HTS triage}

One of the most important aspects of a successful screening campaign is the post-HTS workflow design, or 'screening tree' (Figure 4). A well-designed screening tree helps the project team sift through the hundreds to thousands of HTS actives to prioritize tractable, drug/lead-like compounds for further downstream studies such as determining the mechanism(s) of action, hit-to-lead (H2L) chemistry and higher-order experiments (e.g., cell based, in vivo). Ideally, this should be done as efficiently and rigorously as possible to arrive at a quick 'go/no-go' decision regarding the drug discovery project at hand. At the root level, the triage process has two opposing yet complementary principles: eliminate from consideration compounds with unfavorable properties; and identify the subsets of compounds with the most favorable properties for follow-up testing (we note that the emphasis on each of these principals depends on the

\section{Key terms}

Screening tree: The collection of assays and workflow in an (v) HTS campaign, including pre-HTS steps, such as library design, the HTS itself, and post-HTS triage operations such as counterscreens, orthogonal assays, cross-validating assays, cheminformatics and analytical chemistry.

Tractable/tractability: Hits whose activity has been confirmed using fully characterized compounds that have been synthesized and purified in house. 
Box 1. Evolution of chemical libraries.

My (MA Walters') industrial career at Parke-Davis began in 1997 when I joined its Combinatorial Chemistry group. This was in the middle of the 'combinatorial era' when the Diversomer apparatus was being developed, and the details of scavenger and quench reagents were being reported from the group in Ann Arbor (MI, USA) [31,32]. Over the course of the next 10 years at Parke-Davis (and later Pfizer, Ann Arbor Laboratories) I was involved with library design and synthesis, the CNS therapeutic area and the aforementioned Lead Discovery Group. These were the days of compressed libraries (multiple compounds per well) and the proposed 10 million compound library. I chaired the LIFE (Library Idea and File Enrichment) Committee in Ann Arbor along with Dr Jack Hodges. This committee was charged with the evaluation of ideas for focused library synthesis that would then be outsourced to companies like Tripos (St Louis, MO, USA) and ChemRx (San Francisco, CA, USA). These companies would typically prepare libraries of more than 2500 compounds from each accepted library proposal. These efforts across the Pfizer sites lead to an increase in the corporate library of over 1 million compounds. Prior to these efforts, the corporate library that was screened in Ann Arbor was primarily 'legacy' compounds that were the result of medicinal chemistry programs at the site. After extensive file enrichment, the number of compounds prepared by combinatorial chemistry began to outnumber the legacy collection. This became an important consideration as compounds prepared by combinatorial methods began to turn up in highthroughput screening (HTS) campaigns more frequently. This marked a turning point in the practice of HTS triage. In the early days, probably up to about 2000 compounds produced by combinatorial chemistry at vendors (each given an -R suffix in the corporate database) were not typically purified and biologists often refused to screen these potential mixtures. This - $R$ suffix was later dropped and biologists would then often only reluctantly screen compounds that fell in sequential order as represented by their database numbers. This reluctance to screen compounds prepared in parallel changed when mandatory purity requirements of $>90 \%$ were imposed on all synthesized compounds and when biologists realized that HTS actives from combinatorial libraries could be easily and rapidly followed up in medicinal chemistry. This often reduced the focus on so-called singletons, which were primarily the 'legacy' compounds prepared specifically for medicinal chemistry projects. Coupled with the observation by Baell et al. [33] that many classes of PAINS arise from easily synthesized scaffolds (i.e., 'combiphilic' scaffolds. While at the Lead Discovery Group, the term 'combiphilic' was coined by Dr GL Bolton to described scaffolds and processes that could easily be used in combinatorial chemistry [MA Walters, Personal Data]) one wonders what effect this trend has had on the output of HTS in the pharmaceutical industry and, perhaps more telling, in academic laboratories [Walters MA, Personal Data].

nature of the target, assays and data). Since the determinants of project success are fundamentally linked to chemical structure and its accompanying properties, we believe a chemocentric approach, as opposed to a strictly potency-based approach, is essential for prioritizing chemical matter for follow-up experiments. Naively (in our opinion) choosing compounds based solely on activity can be problematic for several reasons, as it could lead to the selection of artifacts, nondiverse sampling and unfavorable chemotypes in terms of chemical synthesis, physicochemical properties or intellectual property [48]. The major strength of HTS triage is that it can help channel a finite set of resources into the compounds with better chances of real success further along the discovery pipeline. We contend this is best accomplished by: working together with biologists and screeners to design an efficient screening tree and robust assays; incorporating cheminformatic methods to efficiently process and analyze large data sets; and maintaining a chemocentric (medicinal chemistry) approach to data analysis.

Triages are an integral component of nearly every successful screening campaign. Unless every active from an HTS campaign was chosen for follow-up and then subjected to every secondary screen in the post-HTS screening tree, then by definition some type of triage occurred. It is difficult to objectively compare one triage to another, given the subjective components of HTS triage and the nuances surrounding each particular project. Triages could be evaluated for both their overall efficiency and their ultimate ability to identity useful chemical matter. Like many scientific fields, two triage experts may design completely different strategies, both of which may ultimately answer the same scientific question. However, finite resources mean there will always be 'known unknowns' and 'unknown unknowns', and an HTS campaign and its associated triage that fails to identity a chemical probe or drug lead does not necessarily mean the HTS or triage was 'flawed'. As we see it, one weakness of HTS triage is that it is difficult to evaluate the process in a rigorously objective manner, as this would require complete knowledge of a system. However, we point out there are several success stories of HTS triage we believe are particularly informative for readers [49-54]. One trend we notice is that the actual triage processes are not emphasized or described in- 

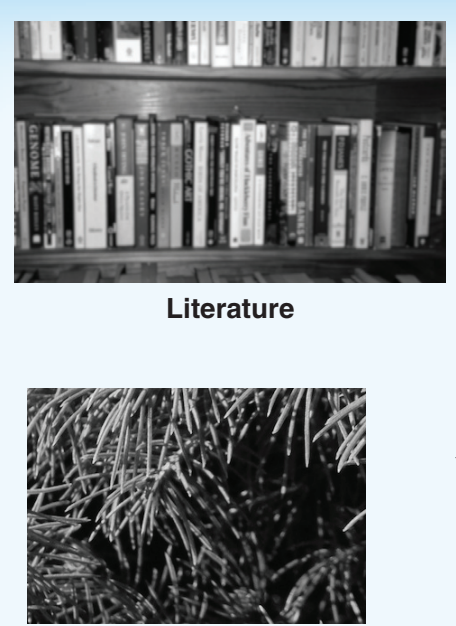

Natural products

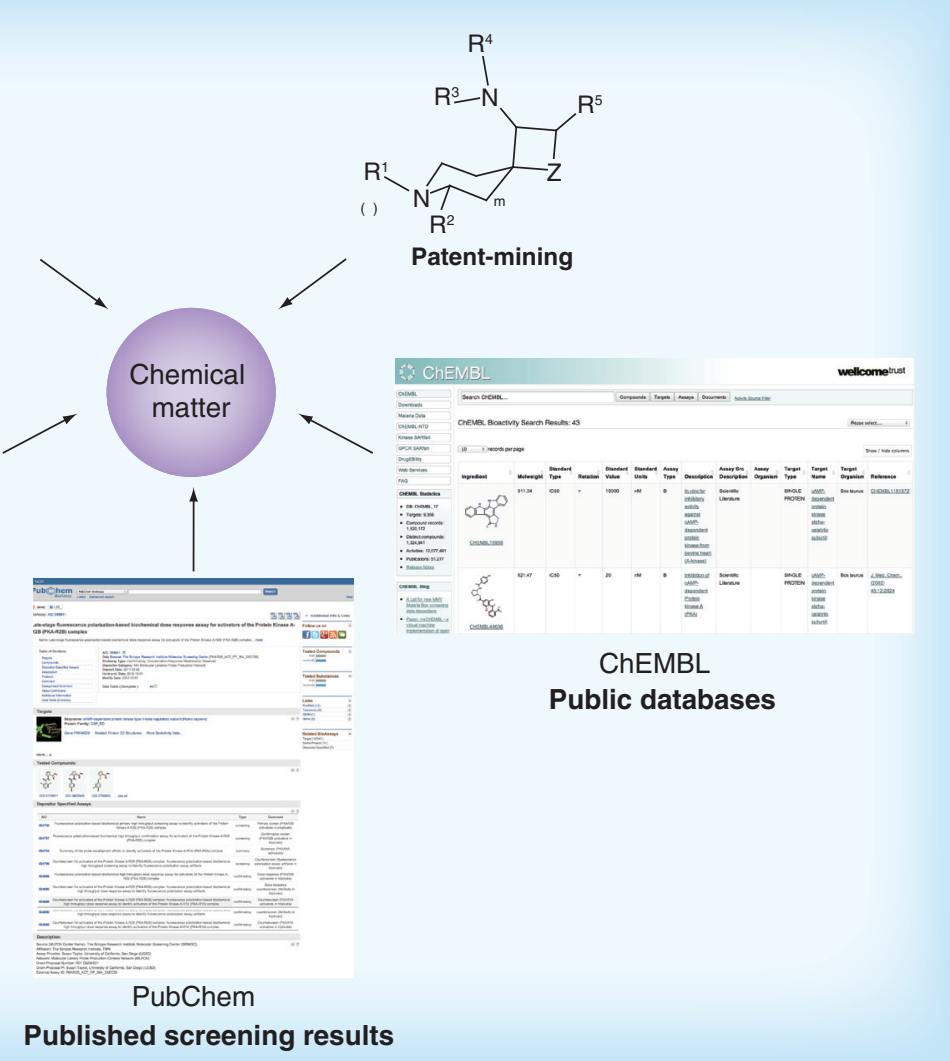

Figure 3. Alternative approaches to chemical matter other than high-throughput screening. PubChem and ChEMBL are examples of publicly available chemical databases [41-43].

depth in the scientific literature [55]. Oftentimes, the details of the triage process are mentioned in passing, or described briefly in supplemental materials. This can make it difficult for readers to evaluate how a project team decided on a particular compound or chemotype for lead optimization, for instance. As we will describe later on, it is often when some critical component of a triage fails or is omitted that the significance of HTS triage becomes obvious.

Depending on the active criteria and size of the screen, HTS campaigns can have hundreds to thousands of primary active compounds, although the actual number of true positives is almost certainly a much smaller subset due to false-positives and assay artifacts. This number can dwindle even further when promiscuous bioactive compounds are triaged. A typical screening tree in either academia or industry consists of post-HTS statistical analyses, cheminformatics analyses, assay-specific counterscreens, orthogonal assays, selectivity counterscreens and perhaps some preliminary mechanistic studies, SAR exploration and cell-based assays (while this arrangement is typical for many cell-free HTS campaigns, we note that many of the same core principles can be applicable to cell-based or phenotypic screens, but are not discussed in more detail here for sake of brevity).
The exact order and composition of the follow-up screens and cheminformatic analyses varies from project to project. Factors such as the target, the size and

\section{Key terms}

Artifact: An assay readout that is not a direct result of the target phenomenon being assayed; often the result of experimental conditions, assay method and/or instrumentation. A straightforward example would be a compound that fluoresces in the same wavelength as the readout of a fluorescence intensity assay.

False positive: An assay readout by a known inactive compound that is erroneously classified as an active compound during the triage process, usually the result of experimental anomalies, poor assay robustness or an artifact source. Nonspecific enzymatic inhibitors like chemical aggregators have been described in the literature as 'false positives', but by strict definition in enzymatic inhibition assays, they are actually true positive inhibitors, albeit by nonspecific and therapeutically uninteresting mechanism(s).

Counterscreen: An assay in which compounds are tested versus a target or assay modification for undesired attributes such as nonselective inhibition (e.g., selectivity) or assay interference (e.g., artifacts).

Orthogonal assay: An assay that interrogates the same biological phenomenon as the primary or HTS assay through an independent assay readout (e.g., a radiolabelled substrate assay to follow-up a fluorescence-based HTS). 
Box 2. Parallel approaches to the development of potential PKA inhibitors.

In a recent project related to the use of PKA inhibitors as potential therapeutics to treat SCA1

(spinocerebellar ataxia 1), we employed each of these methods profitably [44]. There is no evidence that

PKA has ever been targeted by a major pharmaceutical company. While our screen of approximately $200 \mathrm{~K}$ compounds using Caliper technology was being performed (which eventually resulted in six confirmed active compound series, vide infra), we also examined the synthesis and activity of structurally simplified derivatives of the known PKA inhibitor, balanol [45,46]. Additionally, we mined a reported screen for PKA inhibitors in PubChem (Bioassay AID = 524), which yielded a further series for exploration. Finally, we prepared compounds based on numerous Akt programs (Akt [also known as PKB] is a close structural relative of PKA, lying on the AGC branch of the kinome [47]) that had been executed in the pharmaceutical industry. In this case, many potent and selective PKA inhibitors had been made and discarded in the process of optimization of scaffolds as selective Akt inhibitors. We resynthesized and tested some of these compounds following published procedures and eventually developed a collaboration with a major pharmaceutical company. They mined their repository to help us find compounds that would assist us with in vivo confidence-in-mechanism studies. These compounds came primarily from published work, but the hope is that further collaboration will lead to the sharing of unpublished compounds with better potency, selectivity and physicochemical properties [Walters MA, Unpublished Data].

composition of the chemical library, the nature of the HTS assays and screening data, the available resources, project timelines and also the available expertise can greatly influence the order and composition of the screening tree. Typically, some form of cheminformatics and statistical analyses are performed with the HTS data to assist in cherry-picking the primary screening actives (Figures 4 \& 5). From there, follow-up confirmatory experiments and key counterscreens can be performed. As compound attrition mounts and more data is generated for each compound, the surviving compounds can be subjected to higher-level analyses and increased scrutiny. These types of analyses can help select the most promising compounds for purchasing and the more resource-intensive experiments. In our screening trees, we prefer to have a mix of cheminformatics and knowledge-based analyses (e.g., PAINS filters, calculated descriptors, general medicinal chemistry considerations) and actual experimental screens, including some redundancies when resources permit. This is both an acknowledgement to the limitations of performing 'armchair chemistry' and our own preferences for key triage decisions to be supported by several lines of experimental evidence.

One question that is often asked of HTS campaigns is 'who should pick the compounds for follow-up?' We contend that those typically in the best position to prioritize compounds for HTS campaigns are experienced medicinal chemists trained in actual HTS triage, supplemented with input from members of the drug discovery team. This expertise ideally requires a diverse and integrated knowledge base consisting of organic synthesis, medicinal (and analytical) chemistry, ADMET principles, cheminformatics, molecular pharmacology, biochemistry, assay design and biological structures. Despite the wealth of accumulated knowledge regarding HTS and medicinal chemistry, ranking the most tractable compounds is as much an art as a science, as there can be considerable variation in the ranking of compounds among medicinal chemists $[25,26]$. However, without a strong background in synthetic organic and medicinal chemistry, compound selection would presumably be made mostly on activity profiles or other factors that may not be related to or predictive of the chemical or bioactive nature of the compounds themselves.

\section{Data quality \& hand-off can influence HTS triage}

An oft-overlooked facet of the post-HTS process is the data hand-off between HTS and medicinal chemistry. Data mining for less obvious 'hits' (e.g., less potent but more ligand-efficient compounds) is an increasingly utilized strategy [56,57]. A major obstacle to employing this and related strategies with confidence can be data quality. The data being delivered to those in charge of post-HTS triage and follow-up prioritization are inevitably linked to the HTS assay robustness. To mine data with more confidence and to have better odds of success, especially to find compounds that hover near the chosen activity cut-offs, assays with robust Z' factors are preferable as well as other sound statistical descriptors [58-61]. We believe drug discovery teams taking the extra time and effort to optimize HTS assays for even slightly-improved Z' factors and other measures of assay quality can pay dividends down the road, including a reduction in false positives and an overall increase in confidence of the screening data. Data mining can also be enhanced by statistical corrections for the HTS results, which can stem from row and column effects, temporal considerations, and different production runs, to name a few variables [61-63]. Other strategies to increase confidence in screening data are to initially screen compounds in replicates or at multiple concen- 


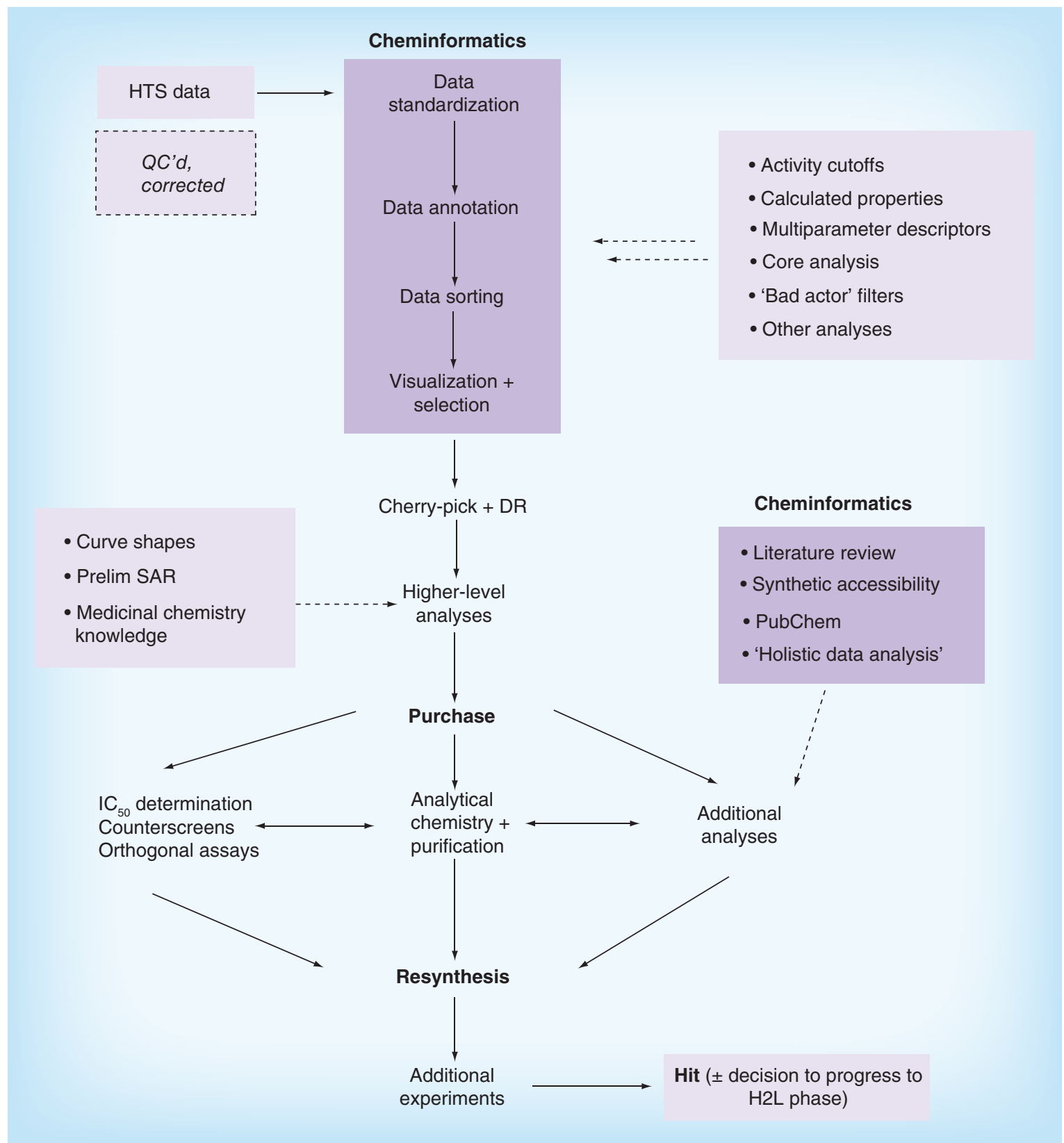

Figure 4. Sample post-high-throughput screening triage screening tree. The process begins with data hand-off from the HTS, from which a series of cheminformatics operations can be performed to standardize, filter and annotate the data. As compounds are progressed, more detailed and time-consuming ('higher-level') analyses and resource-intensive experiments can be performed. We note that the order and details of each operation can vary depending on multiple factors (e.g., target, assay methods, library size and composition, available resources, expertise, project timeline and data volume). For instance, some groups may elect to test all actives by dose-response and counterscreens, and then perform more detailed chemocentric and cheminformatic analyses with the resulting data.

DR: Dose-response; H2L: Hit-to-lead; HTS: High-throughput screening; QC: Quality control.

trations ('quantitative HTS'), although the equipment and other resources needed for these approaches can be prohibitive to many academic groups [58,64]. To ensure the long-term success of an early drug discovery project, we recommend employing sound statistical corrections to the HTS data prior to hand-off to medicinal chemists. To employ such corrections, certain controls, reference compounds and control plates may need to be included throughout the screening campaign, and therefore it is highly desirable to insist and agree on these types of corrections prior to initiating the fullfledged HTS campaign [65]. After screening a large 


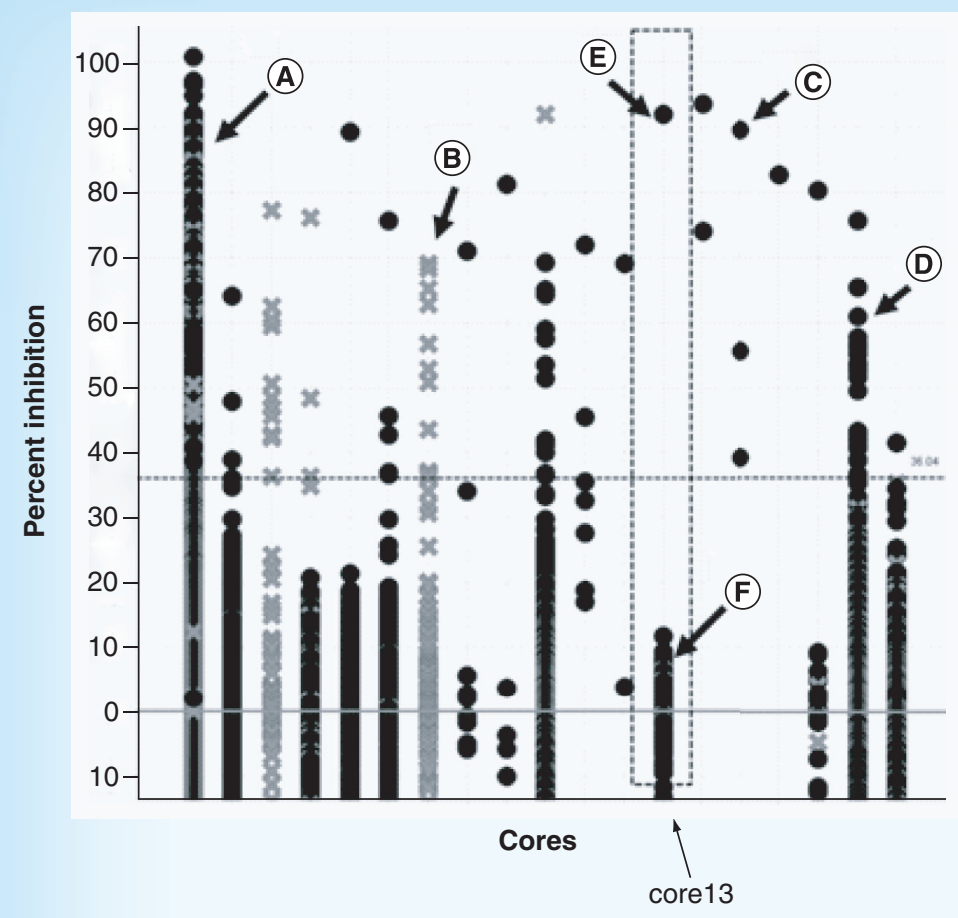

(B)<smiles>O=C(O)c1cccc2c1NC(c1cccc(Br)c1)C1CC=CC21</smiles>

(C)<smiles>O=S(=O)(c1cccc2cnccc12)N1CCCNCC1</smiles>

\section{Scaffold analysis visualization}

- View of very preliminary SAR (e.g., core13)

- View of 'flagged' annotations, such as PAINS (shown here)
Ave $\mathrm{PI}+30$

Non-PAINS compound

PAINS compound

Spotfire $^{\circledR}$ view

(D)<smiles>c1ccc2c(N3CCOCC3)nc(-c3ccncc3)nc2c1</smiles><smiles>COc1ccc(C)cc1NS(=O)(=O)c1ccc2c(c1)CC(C)N2C(C)=O</smiles>

Figure 5. Example of a cheminformatics-assisted core analysis during a post-high-throughput screening triage. See also Box 4. The annotated high-throughput screening data are from a PKA screen performed in the ITDD. (A) Singletons; (B-F) representative compounds from three core classes. (B) is an obvious PAINS (substructure alert bolded). Compound (C) is fasudil, a known kinase inhibitor. Compound (D) has a structure reminiscent of known kinase inhibitors (note that the core containing compound [D] has several examples of active and inactive analogs, a promising early pattern that shows potential for early SAR exploration and argues against many of these compounds being statistical false positives). Core13, which contains compounds (E \& F), has a profile we typically view with skepticism (note that while [E] is deemed active, all remaining compounds in this core are inactive, including the close analog [F]). This example also shows that effective use of modern cheminformatics software allows for large amounts of complex data to be analyzed efficiently. 
number of compounds, portions of compounds are termed active by cut-off criteria, often three standard deviations about the mean percent inhibition (' $3 \sigma$ '), although there are alternative forms of compound selection besides employing strict, sometimes arbitrary cut-offs, but these methods typically require additional expertise in biostatistics $[48,66]$. Usually numbering in the hundreds to a few thousand compounds, this number of actives must be skillfully culled to arrive at a much smaller subset for follow-up experiments. As described earlier in the introduction, this process requires an integrated (and artful) application of statistics, cheminformatics and chemical intuition that is honed by repetition and knowledge sharing.

\section{The good, the bad \& the PAINS}

One of the key roles medicinal chemists can play in the prioritization of chemical matter is the triage of potentially intractable and 'risky' compounds with unfavorable properties. After defining the activity criterion, one of the steps we employ is a series of cheminformatics filters to weed out undesirable substructures or compounds with poor, nonlead-like calculated physicochemical properties. One such step is the computational identification and removal of promiscuous compounds (aka 'frequent hitters' and 'bad actors') such as PAINS [33,67-69]. While several hundred substructures are described in the seminal paper, the chemical mechanism(s) of bioactive promiscuity for many of these scaffolds are uncharacterized or matters of speculation. Scores of these problematic chemotypes have appeared in many of our institutions' HTS campaigns [70,71]. Similar classifications have been reported, such as REOS and others [72], although some of these filters may be too draconian for academic HTS campaigns since they are designed to ensure long-term survival of actives as they progress to preclinical candidates. It is the overwhelming consensus among seasoned medicinal chemists that these compounds (i.e., PAINS and REOS) be avoided for follow-up chemistry or touted as lead compounds or even chemical probes. Despite the awareness of PAINS among reviewers and investigators, compounds flagged as PAINS still manage to make their way into the scientific literature, one of the more notorious and debated examples of these PAINS being rhodanines [73,74]. Medicinal chemists can also flag compounds for potential sources of promiscuous enzymatic inhibition such as thiol-reactive compounds (i.e., electrophiles), redox-active compounds or covalent modifiers by substructure or by first principles. In terms of thiol reactivity, there are several substructure alerts reported in the literature besides the more obvious reactophores (e.g., acid chlorides) [75-78]. Finally, medicinal chemists are optimally suited to spot compounds with poor physicochemical properties that are unlikely to advance far in the discovery pipeline. This filtering can often be done by many commercial software packages, but it is our opinion that having a solid background in hands-on medicinal chemistry (with its accompanying tacit knowledge) can be an important user-provided quality control for the interpretation of such calculated descriptors. For example, substructure filters often flag actives as objectionable even though experience would suggest they are perfectly reasonable. This is a reflection of the challenges of substructure definition, and should be taken into account before throwing out actives without considering the substructure in the context of the whole molecule. Given this, we tend to prefer to 'flag' rather than discard compounds outright. This practice is both a nod to the challenges of using hard cut-offs in an art that should at least be multiparametric in its decisionmaking and to leaving the vial, as it were, open to the examination of chemical matter for tough-targets that typical filters close the cap on.

Chemists and assay designers must work together early on to design screening trees with selectivity, orthogonal assays and counterscreens appropriate for the therapeutic hypothesis. Cost is often a factor. However, the often unexpected costs associated with pursuing promiscuous or artifact-based actives must always be factored into this equation. Selectivity assays are an important component of any post-HTS screening tree, as they can provide clues about the bioactive promiscuity of a given compound. What is a good cut-off for selectivity from a medicinal chemistry viewpoint? While it is possible (but more difficult) to optimize for selectivity starting from a pan-active compound, a good general rule should be greater than five- to ten-fold difference in $\mathrm{IC}_{50}$ values, although the actual threshold depends on the therapeutic hypothesis and expert examination of the target and available data. Often times, $\mathrm{IC}_{50}$ values from independent experiments can vary several fold, so medicinal chemists can help caution against apparent selectivity when there is

\section{Key terms}

Thiol-reactive compounds: Compounds that are capable of forming covalent bonds with biological thiols such as coenzyme A, glutathione and protein cysteine(s) under assay or biological conditions. These compounds are generally avoided in most drug discovery campaigns because of promiscuous bioactivity profiles and ADMET liabilities.

Redox-active compounds: Compounds capable of generating hydrogen peroxide $\left(\mathrm{H}_{2} \mathrm{O}_{2}\right)$ under certain assay conditions (often in the presence of strong reducing agents like DTT or TCEP), which can alter biological processes, usually through oxidation of biological thiols. 


\section{Key term}

Promiscuous inhibitors: Compounds that show apparent bioactivity versus multiple, unrelated biological targets and/or processes, often independent of assay format.

only a few-fold difference in $\mathrm{IC}_{50}$ values between different enzymes. We speculate that the apparent selectivity for many promiscuous bioactive compounds, including many PAINS, may be the different intrinsic susceptibilities of different targets to various forms of nonspecific enzymatic inhibition. For instance, two closely-related enzymes may have different reactivity profiles with respect to a particular electrophilic compound series, which could have the unfortunate effect of leading to a false sense of selectivity that is often invoked as evidence of mechanism-based activity [Dahlin Jl, Walters ma, Personal Data]. Selectivity counterscreens should aim to use some biomolecules closely related to the screening target, especially those that may be predictive of off-target effects. Additionally, it may be useful to assay a completely unrelated enzyme or biomolecule to gauge promiscuity effects. In practice, this may be done by including the candidate compounds in another unrelated HTS campaign. Whenever possible, reference compounds and appropriate controls (positive and negative compound controls) should be included in these assays to judge the quality of the assays and measure inter- and intraexperiment variability. Input regarding the appropriate orthogonal assay and counterscreens is also an important contribution that medicinal chemists can make to decorating the screening tree. The underlying chemical basis of the HTS assay should be carefully considered so that an orthogonal method to cross examine the biological phenomenon can be designed, even at the expense of being lower-throughput, as is often the case with orthogonal assays. There should be some type of correlation between the activity in the primary HTS method and the orthogonal methods, and in our experience it is worth the investment of time and resources to design, optimize and finally corroborate the primary and orthogonal assays before beginning the HTS production run.

Medicinal chemistry concepts can be used advantageously to help design orthogonal and counterscreens to identify assay artifacts and promiscuous inhibitors. Medicinal chemists can be a significant asset to HTS campaigns by triaging compounds with known liabilities such as redox activity, chemical aggregation and thiol reactivity (some specifics of problematic substructures will be discussed in the next section). Compounds that produce $\mathrm{H}_{2} \mathrm{O}_{2}$, often fueled by the presence of reducing agents such as DTT and TCEP in many biological buffers, can nonspecifically inhibit enzymes or alter biological processes. This can occur by oxidizing protein cysteines or other critical amino acid side chains, for instance, and can mimic a well-behaved inhibitor [79]. However, we have found the use of a facile counterscreen such as the HRP-PR assay can easily identify such compounds for triage [80,81]. Compounds that nonspecifically inhibit protein activity by chemical aggregation can also be identified by several different counterscreens, such as a surrogate $\beta$-lactamase assay (our current assay of choice), Hill slope analysis, DLS or NMR-based methods. Medicinal chemists should emphatically insist that HTS assays and any follow-up experiments include detergents (unless prohibited by experimental considerations) to minimize the chance of enriching chemical aggregators [70,82-90]. Another area where medicinal chemists can greatly assist the HTS process is the triage of thiol-reactive compounds, which can nonspecifically react with protein thiols and lead to promiscuous enzymatic inhibition and potentially toxicity [78]. These compounds (or certain metabolites) can also react with other biological thiols such as glutathione and coenzyme A, all of which can have important ADMET implications. Multiple experimental methods are available to probe thiol reactivity, including glutathione-based competition and HPLC-based methods, NMR, fluorescent probes, peptide probes and ALARM NMR/LC-MS [78,91-97]. Other sources of promiscuous inhibition or assay artifacts include chelation, fluorescence, fluorescence quenching, singlet-oxygen production or capture, compound-reporter interactions (e.g., luciferase reporters) and apparent compound-antibody interference [85,98-110]. We note that many of these mechanisms such as redox activity and thiol reactivity are particularly relevant to many cell-based assay readouts, including cell proliferation and reporter systems [Personal Data]. Medicinal chemists can strongly influence the identification of such compounds by correlating the structures of HTS actives with the predicted chemical mechanism(s) of assay interference. Finally, promiscuous compounds can be checked by careful literature and database searches, including PubChem and SciFinder [111-113]. It is our experience that the screening tree must be in place as early in the biology-chemistry HTS collaboration as practical. Strict decision points should be established and enforced. In our observations, projects sometimes build up so much momentum that it is difficult to admit defeat. At that point it is tempting to invoke the faulty logic of sunk-costs and continue down a less-than-fruitful pathway.

Detailed inspection of raw dose-response data is yet another layer of the triage process of target-based assays. Besides simply examining the $\mathrm{IC}_{50}$ values derived from curve-fitting regressions, we find additional value in examining the shape and quality of the 
curves [114]. Such analysis can provide important clues about the nature of chemical inhibition and data quality. Dose-response curves can be grouped into categories by quality of the curve fit to the data, efficacy and the number of asymptotes [64]. Such descriptors can help prioritize compounds with full dose-responses, for instance. Hill slope analysis is another important consideration, as it can give clues about potential mechanisms (e.g., positive and negative cooperativity) [115]. During one of our recent post-HTS triages, steep Hill slopes were also useful for flagging potential chemical aggregators [70,116]. The consideration of dose-response data can be incorporated at several points in the triage process, depending on when compound titration experiments are performed, the specifics of the HTS organization and the number of compounds to be analyzed. In practice, we have found it useful to display curve descriptors alongside the $\mathrm{IC}_{50}$ values once dose-response data are available and to triage compounds with steep Hill slopes prior to more detailed cheminformatics analyses. We find the more nuanced analyses of dose-response data is increasingly useful as the number of compounds to be considered is whittled down by the triage process.

\section{The role of cheminformatics in HTS triage}

The cornerstone of cheminformatics in HTS is the ready availability of digital structure data associated with digital compound attribute data. This combination of data allows the rapid handling of millions of data points and calculation of physicochemical properties, similarities, shapes, substructures, and so on to go along with activity data that have been collected in the HTS. This compound attribute data can then be used to generate information (inference of relationships) and finally, knowledge, the understanding of what these relationships mean as applied to the task at hand. Cheminformatic methods also enable the rapid searching of the scientific and patent literature by chemical structure (Box 3), a critical but underutilized tactic that we will describe later. As throughput continues to increase, and as data and analyses become more complex, there is always the possibility for information overload to stymie HTS triage - especially as more data accumulates throughout the course of a multistage triage. However, coupled with a well-designed screening tree, modern cheminformatic tools and vigilant data management can enable large data sets to be quickly processed and analyzed effectively by experienced users.

There is a large body of literature available on the application of cheminformatics to HTS triage [118-122]. Essentially, cheminformatic methods help the medicinal chemist rapidly sift through the large number of active and inactive compounds that arise in a typical HTS (Box 4). On large data sets, this work usually consists of the calculation of physicochemical properties (e.g., molecular weight, clogP, rotatable bonds, among others) [24,123-125], ligand efficiency metrics (a combination of properties and activity) [126-130], structural attributes [33,72,131-132] and annotation of the HTS data set with these values or flags. This is typically followed by strict cut-off filtering (e.g., only compounds with a molecular weight $\leq 500$ are kept), multiparametric filtering [133,134] or flagging of the actives (or a combination of these methods) to help prioritize and reduce the number of compounds that need to be considered further. It is imperative that similar schemes be applied either pre- or post-vHTS since in these cases bioactivity is not used as filter until after the bulk of the chemical matter has been selected [39,135-137].

\section{The contribution of purification $\&$ analytical sciences to HTS triage}

Another critical (but often overlooked) contribution of medicinal chemistry in the post-HTS screening tree involves hit confirmation by analytical chemistry techniques and chemical synthesis. After confirming dose-response with library samples, typically the top

\section{Box 3. Structural landscapes.}

In the mid-2000's I (MA Walters) proposed to download structures from project-related articles and patents that could be found in the Chemical Abstracts Service (CAS) [177] database and then use cheminformatics to analyze this data. A group of us (including Dr Chris Kibbey, Pfizer Scientific Computing) envisioned that visualization of the structural landscape encompassed by compounds in the literature related to our chosen target could lead to a rapid understanding of the medical chemistry explorations that had preceded our work. As it is still the state of affairs, downloading and exporting large numbers of structures from CAS at that time was impossible. In fact, it became apparent that the power of digital structural information tied to literature knowledge was highly underappreciated. We were eventually able to download approximately $10 \mathrm{~K}$ structures (at approximately US\$1 per structure) and view a project chemical landscape where links to the literature were associated with structure, and structures were grouped by similarity. This project eventually died from lack of senior management support, although apparently similar ideas did surface elsewhere within the organization, as structures from patents were used to gauge the relative importance of claimed compounds [117]. 


\section{Key term}

Structure-interference relationship (SIR): The

misguided correlation of chemical structure with supposed biological activity, when in fact the presumed bioactivity is actually a composition of assay interference, false-positives and/or artifacts.

prioritized compounds are supplied by commercial vendors. This is all but guaranteed by the nature of the library employed in screening. Depending on the resources and synthetic expertise on-hand, one can also choose to resynthesize the top compounds in-house. The decision to synthesize in-house or purchase compounds is typically a time versus money issue that must be balanced by the goals and resources of the project team. Most screening compounds are available in milligram quantities from international vendors. The amount of compounds chosen for resupply, budget issues, and types of experiments and quality-controls all dictate the amount of compound needed for resupply. In our experience, we order 10-25 mg of compound, which is enough for making several aliquots for multiple independent experiments and internal quality controls. Typically, most screening compounds from reputable vendors can be supplied within 2-4 weeks. Close structural analogs can also be purchased at the same time to perform a focused SAR ('SAR-by-commerce'). One caveat is that if the choice of actives to pursue is unwise, this may actually degenerate into a structure-interference relationship study that is related to SAR but doesn't really measure target engagement [104].

In our collective experience, there are many times where the vendor-supplied structure is not compatible with the spectroscopic data. Additionally, compounds can isomerize, degrade or oxidize during their shelflife or during the course of an experiment [138-141]. For this reason, careful sample and batch tracking by barcoded vials and an electronic database is very useful. Another related issue is the hydration of compounds stored in dimethyl sulfoxide (DMSO) and the effect of freeze-thaw cycles [142-144]. We strongly recommend re-checking vendor-supplied samples for purity (e.g., HPLC-MS) and structural characterization $\left({ }^{1} \mathrm{H}\right.$ and ${ }^{13} \mathrm{C}$ NMR) $[145,146]$. Ideally, this would be done for every commercial re-supply, but in practice this is difficult for smaller academic labs, and therefore we recommend this be a priority for those compounds that have high probabilities of being recommended for mechanism-of-action studies or analog expansion. The purity of any compound, commercial or synthesized, is nontrivial. In our experience, most vendor-supplied screening compounds have approximately $95 \%$ purity according to ultra performance liquid chromatogra- phy-mass spectrometry (UPLC-MS) analyses [Francis S, Unpublished Data]. However, compound samples with even trace impurities can lead project teams astray. These impurities can be remnants from the chemical synthesis, including transition metal catalysts, leftover starting materials, reactive intermediates, oxidative products, rearrangements or enantiomers, to name a few [146-153]. Therefore, medicinal chemists should insist on in-house HPLC purification (or comparable method) of any compound making its way into critical experiments. Often commercial samples are sold as racemic mixtures, so care should be taken to isolate the active enantiomer responsible for biological activity, such as the use of chiral chromatography or recrystallization, and eventually confirm with an enantiopure sample. Typically, little, if any, information is provided by a chemical vendor about the synthetic scheme of a compound, and it is rather difficult to predict or identify any bioactive impurity a priori. Therefore, after vendor resupply and subsequent HPLC-purification, a third step we employ in the chemical resynthesis and complete characterization of the top compounds [98]. This safeguard ensures the compound identity, purity and bioactivity of a potential lead compound, and gives clues about its synthetic feasibility, scale-up and analog expansion.

\section{A demand for compound natural histories}

Lastly, we propose that during hit and active followup it is essential to investigate the 'natural history' of prioritized compounds. This is not only to keep in mind the potential for intellectual property (IP) in the future, but to understand the potential for off-target reactivity, promiscuity and interference. Because all of these are so intimately related to chemical structure, this process is best done by a scientist with an appreciation for medicinal chemistry. Chemical filters and rules can be used to help reduce the number of compounds screened and to prioritize actives for future follow-up. A 'natural history' examination can use the same rules and filters, but also should include searches of relevant data, information and knowledge bases. An example of this is shown in Table 2. Five common core structures were selected based on compounds we have seen repeatedly in assays against a variety of targets. The natural history shows the results from searching these compound classes in SciFinder Scholar (relevant to target, IP, promiscuity, and interference), compound libraries (eMol, an academic library, and the MLSMR), and PubChem. These compound classes have also been flagged as PAINS. Nevertheless, note that these compounds continue to be reported in manuscripts purportedly linked to biological activity, calling into question the validity of these research reports. 


\section{Box 4. Cheminformatics in early high-throughput screening triage.}

- A careful analysis of an high-throughput screening (HTS) will help the project team focus on what are most the attractive actives and can help find compounds that have good ligand efficiency. This analysis typically involves four steps: data standardization; property annotation; substructure sorting; and visualization and selection. The complexity of this analysis is typically proportional to the number of compounds assayed. For typical full-file screens (all of the compounds in a collection), we employ the following procedure. We obtain a structure-data file from our screening group that also contains our unique compound identifier (GPHR number) and the percent inhibition (or other readout) from the primary assay. This structure-data file contains data for all of the compounds screened. This data file is first standardized with respect to molecular representation, duplicate removal, among others, and then annotated with physicochemical property descriptors (clogP, molecular weight, number of $\mathrm{H}$-bond donors, number of $\mathrm{H}$-bond acceptors, topological polar surface area), a pan-assay interference compounds (PAINS) flag ( $0=$ no; $1=y e s)$, a Lipinski filter flag, and the PEI (percent inhibition/molecular weight). We use a combination of Pipeline Pilot and Canvas to perform this annotation, but other software can also be employed. We then examined the top 100 actives (by percent inhibition and then by PEI) looking for common core structural motifs [11,120]. These motifs are used to create substructure filters for these cores. This core generation can be done manually or with software like Canvas. Finally, all compounds, both actives and inactives, are annotated with a core designator (core1, core2, core3, core [n]) or with a singleton designator (typically $n+1$, where $n$ is the number of cores found in the first pass analysis of common cores. No numerical filtering is performed at this point. This comprehensive data package is then examined using Spotfire Lead Discovery and the best compounds are prioritized for cherrypicked IC $C_{50}$ determination from the original screening plates. Activity is typically defined as having a percent inhibition $\geq 3 \sigma$ from the average activity found in the assay, although this cut-off can be varied depending on the assay and other factors. In our experience, percent inhibition is subject to large errors. However, we consider it a confirmation of preliminary activity if several compounds containing the same core structure appear as actives in our first analysis. Our first-pass prioritization is based on a consideration of activity and a balance of calculated physicochemical properties as described elsewhere. All PAINS compounds are deprioritized unless our experience suggests that they have been flagged inappropriately.

- An example of this type of analysis is shown in Figure 5. An HTS targeting the discovery of PKA inhibitors was performed on our screening library (at that time $196 \mathrm{~K}$ compounds) and gave an initial data file that included 470 actives as defined by a $\geq 3 \sigma$ criterion from the average activity ( $0.2 \%$ actives). The top 100 actives were deconstructed into core structures and each compound in the HTS data set was assigned to a core. Core structures were found in 17,741 structures in the HTS ( $9 \%$ ) while 87 of the 470 actives were in core structures (19\%). PAINS core structures containing compounds like B were included here for illustrative purposes. Structures like $C$ and D confirm that the assay was selecting compounds of interest. Compounds for follow-up would be selected from cores that showed multiple compounds above the 'active' cut-off (highest priority), and both the singletons (381/470 actives) and compounds with relatively high PEI would be considered for dose-response confirmation. Compounds such as those from core13 are viewed with skepticism. Notice that while compound E is active, all of the related analogs in this series are inactive, including many close structural analogs such as compound $\mathrm{F}$.

- Following cherry-picked IC $\mathrm{C}_{50}$ determinations and perhaps other follow-up assays, compounds exhibiting sigmoidal-shaped dose-response curves and having $\mathrm{IC}_{50} \leq 30 \mu \mathrm{M}$ are usually prioritized for repurchase using the same criteria discussed above. Typically, $10 \mathrm{mg}$ of every prioritized, commercially available compound is purchased (some close analogs may also be purchased to help establish preliminary SAR), and these samples undergo quality-control tests (LC/MS/PDA/ELSD) to help establish identity and purity, and is then retested. Quality control will often include compound purification, as it is becoming increasingly clear that some assays are very sensitive to trace impurities (e.g., metals, electrophilic reagents, and decomposition products) in the commercially available samples. Additionally, NMR methods may be used to confirm identity in some cases. Confirmation (IC $\leq 30 \mu \mathrm{M}$; sigmoidal-shaped dose-response curves; $0.8 \leq$ Hill slopes $\leq 1.2$ ) of these more well-characterized compounds in the primary assay helps instill confidence that the selected chemical matter is worth spending more time and money investigating during both the hit- and series-validation stages of the project. As a matter of course, we annotate the entire data package with information for each compound as it is generated, and continually review this data to insure prioritized compounds are properly followed-up.

- Larger data sets of actives from HTS that are found in industry will typically require a more detailed set of filters (absolute or multiparametric) to whittle them down to a manageable size. In our experience, the number of actives from a typical academic library full-file screen is $<1 \mathrm{~K}$ and highly automated triage is not necessary. Often this number can be quickly culled after cherry-picking, dose-response confirmations and some key follow-up screens. At this point in the triage process we also perform fairly extensive literature and database searches to determine the natural history of our best series. The expansive amount of data available on some commercially available compounds can be overwhelming. However, we find that a search of Scifinder (using either exact or similarity searches) can often offer us a quick readout on a compound series. For example, the number, document type, and abstract of the available references can often allow us to bin series with respect to their priority for follow-up.

Natural histories like this can further prioritize compounds for follow-up, allowing the most efficient use of limited resources. Such searches may also turn up previous medicinal chemistry follow-up on the same structural classes, saving the time that could be lost following the same SAR.

A recent publication underscores the importance of performing some detective work prior to reporting or 


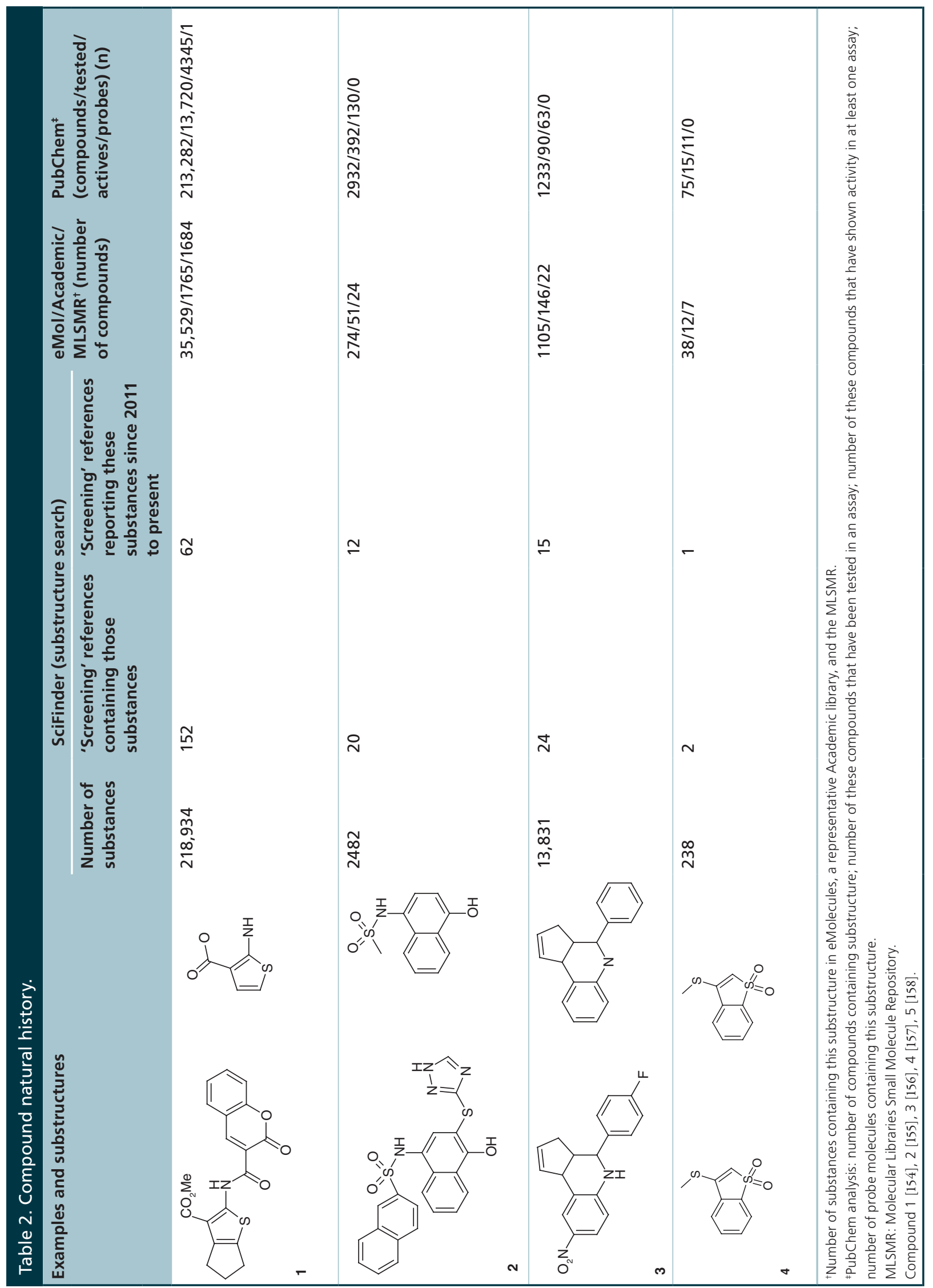




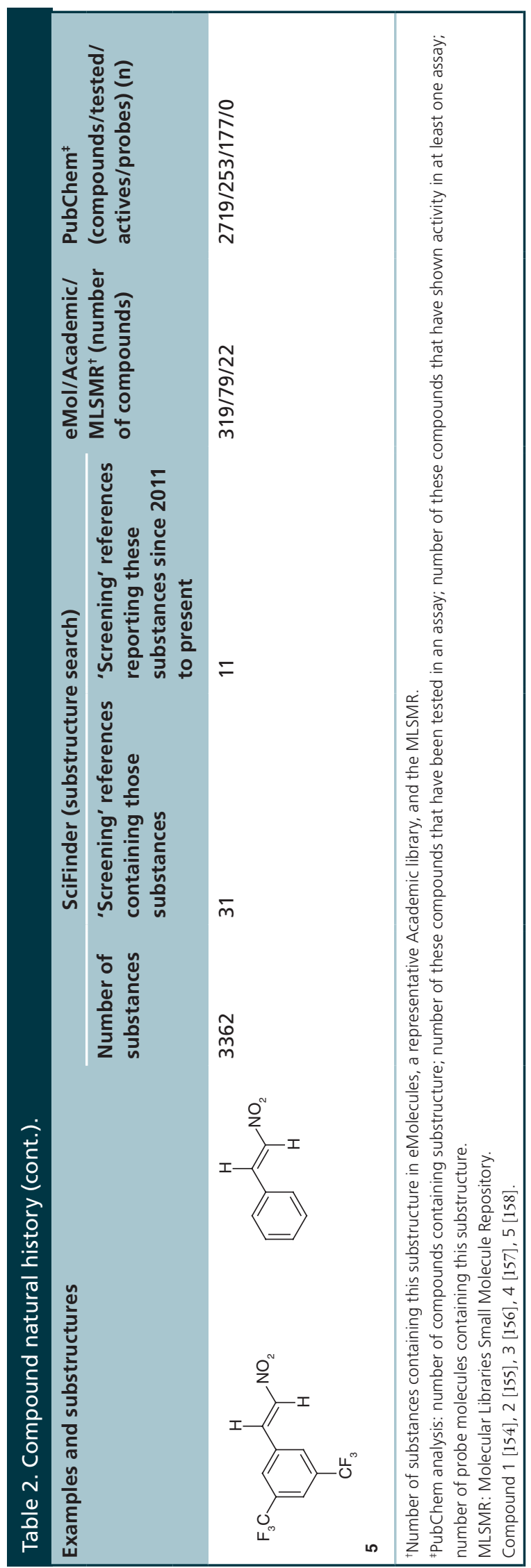




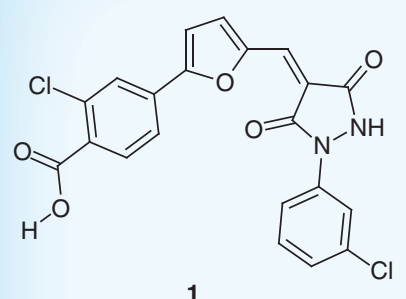

1

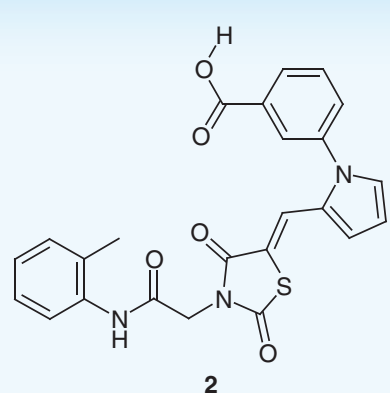

2

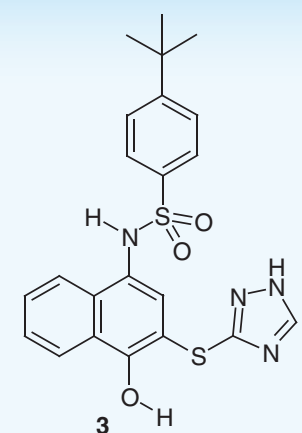

\begin{tabular}{|l|c|c|c|c|r|}
\hline Compound & Exact & Sources & $\geq 90 \%$ & Biol & Ref. \\
\hline $1(\mathrm{~S} 6)$ & 0 & 4 & 79 & 5 & 1 \\
\hline $2(\mathrm{~S} 40)$ & 1 & 4 & $480-164]$ \\
\hline $3(\mathrm{~S} 47)$ & 0 & 4 & 19 & 5 & {$[165]$} \\
\hline $4(\mathrm{HS} 26)$ & 0 & 10 & 26 & 6 & {$[168,169,170,171,172,173]$} \\
\hline
\end{tabular}

Figure 6. The natural history of PAINS compounds reported in a recent manuscript. Searches performed in SciFinder. $\geq 90 \%$ : Compounds that are greater than $90 \%$ similar; Biol: Compounds that were reported in a 'biological study' from a journal article [155,159-172]; Exact: Exact substructure found; Sources: Commercial sources of exact compound.

otherwise following up on HTS results. In this manuscript, the compounds 1-4 are cited as the 'most potent compounds' with respect to inhibiting Keap1-Nrf2 interactions (Figure 6). These compounds were identified by structure-based virtual screening and their activity was confirmed in standard biological assays. Compounds $\mathbf{1}, \mathbf{3}$, and $\mathbf{4}$ are classified as PAINS and $\mathbf{2}$ is closely related to a PAINS substructure. This is not acknowledged in the manuscript.

Admittedly, in the past not all researchers had access to software capable of screening for PAINS substructures. However, almost all authors and reviewers should have access to the CAS database and there are now freeware versions of PAINS filters available [68]. Figure 5 shows an analysis of these compounds in SciFinder, software that is readily available at our institutions. We performed a simple literature 'screen' on the exact structure of each 'most potent compound.' Although each of the compounds 1-4 is commercially available from several vendors, there are $0-1$ references of any kind mentioning the exact structures shown. This, and the names of the vendors selling these compounds, suggests that they were all prepared for sale in screening libraries. In and of itself this is not a problem, although the fact that they have received such scant interest in the literature may be a warning sign. What about related compounds? Have they been reported in the literature? We used an arbitrary cut-off of $\geq 90 \%$ similarity (equivalent to a regioisomeric shift of an aromatic substituent) to look for closely related compounds. The number of substances we found for $\mathbf{1 - 4}$ is shown in the $\geq 90 \%$ column. To better place these substances into context, we then retrieved all of the references for these compounds that were classified by the term 'Biological Study' and refined this list by the 'Document Type' equivalent to 'Journal'. The number of references retrieved by this protocol is listed in the Biol column of the figure. Each of the references is listed in the references column. These references typically report the activity of the closely related compounds against other targets, and some describe analog synthesis resulting in extensive apparent SAR.

None of these references appear in this recent publication. Moreover, reference [168], which we found in this relatively conservative exercise in SciFinder, is the seminal manuscript by Soares et al. that describes the potential for redox interference by compounds essentially $(\geq 90 \%)$ identical to 3 and $\mathbf{4}$. This apparent disregard or lack of knowledge of the preceding literature is not an isolated phenomenon. The two other most recent publications, references [165] and [169], make no mention of PAINS and no mention of the work on similar compounds reported in references [155,165-168] and [167-172], respectively (admittedly, reference [169] does reference an earlier paper by that describes the protocol for detecting compounds capable of redox interference [81]). 
While the knowledge of PAINS substructure might not yet be part of the lexicon of information readily available to researchers, some relatively straightforward literature exercises may have led the researchers to contemplate the potential polypharmacology of their 'hits' and might have raised red flags with respect to compounds 3-4. This exercise in 'natural history' should be done when clusters or series of actives are discovered during HTS triage and well before SARby-commerce or extensive assays resources are devoted to the project. In our opinion, a rigorous analysis of the literature can influence where resources can most effectively impact the ultimate success of a project, be they for selecting which are the best series for follow-up, development of counterscreens, or making a go/no-go decision. It is also worthwhile mentioning that the aforementioned triage best practices and screening trees we describe would have, in all likelihood, flagged these problematic chemotypes at several stages. To us, these case studies, and many others like them where highly problematic compounds are published in reputable journals, further argue for the essential roles of chemistry in HTS triage.

\section{Conclusion \& future perspective}

HTS is an important component of drug discovery. However, we believe that the literature will continue to be overrun by questionable 'hits' until the art and science of chemistry-driven compound triage is more widely practiced and appreciated. This art requires a constant review of the screening literature, knowledge-sharing among those in the field, and triage experience. This science sits on the three-legged stool whose legs are medicinal chemistry, cheminformatics and the purification sciences, and benefits from input from biostatisticians, biologists, screening teams and molecular pharmacologists. In our opinion, the practice of HTS triage has improved over time with: the realization that activity is not the only piece of data that should be considered; the focus on more ligandefficient hits and the understanding that smaller may be better for starting points; and the increased accessibility and sophistication of cheminformatic resources. The field should continue to improve with regards to efficiency and the ability to prioritize tractable chemical matter as: HTS becomes even more accessible, screening throughput increases, assay methods and screening libraries evolve, and more screening data are deposited in databases for data mining and knowledge-sharing. This availability of more screening data will hopefully drive the development of more predictive models of favorable (and also unfavorable) chemical properties, thereby positively enhancing the cheminformatics capabilities of HTS triage. Roadblocks to progress in HTS triage will be the continued availability of PAINS and other nontractable chemical matter in vendor and screening libraries, and what we see as a continued lack of awareness of these nefarious compounds among many medicinal chemists and screeners. This will only improve if the customers - screeners and medicinal chemists - stop purchasing and publishing these types of compounds.

We believe the future can be positively shaped by at least three straightforward mandates regarding

\section{Box 5. The Institute for Therapeutics Discovery and Development.}

There is a long and rich history of academic drug discovery and development in the academic setting. The University of Minnesota (UMN; MN, USA) shares in this rich history, most notably by the discovery of Ziagen ${ }^{\circledR}$ (ViiV Healthcare, Brentford, Middlesex, UK), an anti-AIDS compound that has prolonged millions of lives and brought the university over US\$600 million in royalty payments over the past 15 years. That compound was discovered by Dr Robert Vince, based on a rational drug design approach [175]. His Center for Drug Design remains a key player in the drug discovery at the UMN. Seven years ago, the university decided to embark on an experiment that would involve the organization of drug discovery in an institute that would serve all of the biomedical researchers at the UMN and also support translational projects developed by our close collaborators at the Mayo Clinic. The Institute, which is directed by $\mathrm{Dr}$ Gunda Georg, is comparable to a mini-biotech in its capabilities, and to a small pharmaceutical company in the scope of the projects it engages. The projects primarily arise from the biomedical laboratories at the UMN, but projects from other institutions and industry are becoming more prevalent in our portfolio. The Institute consists of five core groups: HTS, Lead and Probe Discovery (LaPD), Medicinal Chemistry, Chemical Process Discovery and Development (including GMP synthesis), and Pharmacology. Each of these groups is lead by a director with industrial experience. The capabilities of the Institute have led to the purposeful organization of other drug discovery and development groups within the confines of the UMN and an overarching interest at the university of accelerating bench-to-bedside processes (the capabilities of the ITDD have recently been more extensively reviewed [176]). The LaPD is intentionally modeled after the aforementioned industrial-based Lead Discovery Group. We have expertise spanning medicinal chemistry, cheminformatics and purification services, and routinely handle the triage of three to five high-throughput screening campaigns per year. 
publication of assay results: that assay development manuscripts be required to provide evidence of target engagement; that PAINS and other interference compounds be appropriately referenced and flagged; and that authors provide relatively straightforward 'natural histories' on all reported active compounds including a discussion of the relevance of these natural histories to the screening method and the target under investigation. The recommendation that authors upload machine-readable structural information (e.g., SMILES) of reported bioactive molecules will facilitate the initial review and later analysis of these manuscripts [173]. We recommend that all journals consider requiring this as supporting information. Another related recommendation is the inclusion of more detailed descriptions of the post-HTS triage process in manuscripts describing HTS results. This should allow for more critical examinations of the science by readers and call much-needed attention to this critical component of successful HTS campaigns. Such recognition may spur further advances in the field, such as the development of new tools and data/evidence-driven methodologies to render the triage process more effi- cient and successful in terms of identifying tractable chemical matter.

Of course, while the application of these mandates will do much to assure the quality of the science reported, care should be taken to recognize and acknowledge the importance of covalent or irreversible inhibitors, since these will often fail simple and simplistic cheminformatic filters [174]. Should these types of compounds be reported, the relevance of such inhibitors to the target in question should by adequately rationalized. We have no doubt that HTS will continue to provide new starting points for discovery of biochemical probes and drugs. The best way to insure that this occurs in the most effective manner, we believe, is through a partnership between HTS and chemistry that begins early in the discovery process (Box 5).

\section{Acknowledgements}

JL Dahlin gratefully acknowledges the Mayo Clinic librarians for assistance in obtaining scientific literature and Dr Zhiguo Zhang of the Mayo Clinic for mentorship. MA Walters acknowledges all members of the former Lead Discovery

\section{Executive summary}

Medicinal chemistry in high-throughput screening organization and assay design

- Chemists and high-throughput screening (HTS) personnel must work together to obtain high-quality and robust HTS data. Appropriate control plates and reference compounds to correct for systematic errors arising from the screening method can help in this process.

- Plans for confirming bioassay activity should be established early by a combination of assay-specific counterscreens, orthogonal assays and selectivity assays.

- Virtual HTS projects should form early partnerships with biological scientists and medicinal chemists experienced with assay design and post-HTS triage, respectively.

Post-HTS triage

- Post-HTS triage combines the elimination of risky hits (false-positives, artifacts, frequent hitters and unfavorable physicochemical properties) and the prioritization of more promising hits for follow-up experiments.

- Post-HTS triage combines elements of cheminformatics, medicinal chemistry and the purification sciences (a 'chemocentric' approach).

- The evaluation of chemical 'beauty' (e.g., HTS triage) is both an objective and subjective process.

- Potential active compounds should be evaluated for bioassay promiscuity and natural history by conducting thorough literature, database and patent searches.

- Post-HTS triage should include assays to eliminate redox-active compounds, chemical aggregators and promiscuous thiol-reactive compounds, even if these nefarious mechanisms are not initially suspected.

- The structural identity and purity of promising bioactive compounds should be confirmed, and bioactivity verified by chemical re-supply and independent chemical synthesis.

Recommendations for reporting HTS results

- Compounds flagged as pan-assay interference compound should be pursued with extreme caution, and should be considered by reviewers and researchers alike as unsuitable as leads or chemical probes unless proven otherwise by rigorous experimentation.

- More drug discovery teams should publish examples of assay artifacts or 'no-go' decisions, to serve as lessons to others and to prevent wasted follow-up by independent groups.

- Detailed descriptions of the post-HTS triage process should be included in manuscripts reporting novel chemical matter derived from screening campaigns. This should raise awareness of this critical component of successful drug discovery campaigns, increase overall scientific transparency, and hopefully inspire the development of more data/evidence-based triage practices. 
Group (Ann Arbor; MI, USA). JL Dahlin and MA Walters acknowledge the members of the LaPD and ITDD for helpful discussions.

\section{Disclaimer}

The funders had no role in study design, data collection and analysis, decision to publish, or preparation of the manuscript. The opinions or assertions contained herein belong to the authors and are not necessarily the official views of the funders. The content is solely the responsibility of the authors and does not necessarily represent the official views of the NIH.

\section{Financial \& competing interests disclosure}

This work was supported by the Minnesota Partnership for Biotechnology and Medical Genomics (\#73-01), the NIH, the Mayo Foundation for Medical Education and Research and

\section{References}

Papers of special note have been highlighted as:

- of interest; $\bullet$ of considerable interest.

1 Frye S, Crosby M, Edwards T, Juliano R. US academic drug discovery. Nat. Rev. Drug Discov. 10, 409-410 (2011).

2 Gurvich VJ, Byrn SR. NIPTE: a multi-university partnership supporting academic drug development. Drug Discov. Today 18(19-20), 916-921 (2013).

3 Rotella DP. Drug discovery 2012 and beyond. ACS Med. Chem. Lett. 3(3), 172-173 (2012).

4 Tralau-Stewart Cathy J, Wyatt Colin A, Kleyn Dominique E, Ayad A. Drug discovery: new models for industry-academic partnerships. Drug Discov. Today 14(1-2), 95-101 (2009).

5 Tralau-Stewart C, Low CMR, Marlin N. UK academic drug discovery. Nat. Rev. Drug Discov. 13(1), 15-16 (2014).

6 Iserson KV, Moskop JC. Triage in medicine, part I: concept, history, and types. Ann. Emerg. Med. 49(3), 275-281 (2007).

7 Moskop JC, Iserson KV. Triage in medicine, part II: underlying values and principles. Ann. Emerg. Med. 49(3), 282-287 (2007).

8 Swamidass S, Bittker J, Bodycombe N, Ryder S, Clemons P. An economic framework to prioritize confirmatory tests after a high-throughput screen. J. Biomol. Screen. 15(6), 680-686 (2010).

9 Bickerton GR, Paolini GV, Besnard J, Muresan S, Hopkins AL. Quantifying the chemical beauty of drugs. Nat. Chem. 4(2), 90-98 (2012).

10 Lowe JA III. The role of medicinal chemistry in drug discovery. In: The Art of Drug Synthesis. Johnson DS, Li JJ (Eds). John Wiley \& Sons, NJ, USA (2007).

11 Yang X, Parker D, Whitehead L et al. A collaborative hit-to-lead investigation leveraging medicinal chemistry expertise with high throughput library design, synthesis and purification capabilities. Comb. Chem. High Throughput Screen. 9, 123-130 (2006).

- Excellent example of the importance of forming an early medicinal chemistry-high-throughput screening (HTS) the Minnesota Supercomputing Institute. JL Dahlin was supported by the NIH Medical Scientist Training Program at the Mayo Clinic (T32 GM065841), an NIH predoctoral fellowship (F30 DK092026-01), a Pharmaceutical Research and Manufacturers of America Foundation predoctoral pharmacology/ toxicology fellowship and the Mayo Foundation. Some of the research reported in this publication was supported by the National Institute of Neurological Disorders and Stroke (NINDS) of the NIH under award number ARRA-NS04566707S1. The authors have no other relevant affiliations or financial involvement with any organization or entity with a financial interest in or financial conflict with the subject matter or materials discussed in the manuscript apart from those disclosed.

No writing assistance was utilized in the production of this manuscript.

partnership that utilizes many of the best practices described in this article.

12 Leeson PD, Oprea TI. Drug-like physicochemical properties. RSC Drug Discov. Ser. 13, 35-59 (2012).

13 Leeson PD, Springthorpe B. The influence of drug-like concepts on decision-making in medicinal chemistry. Nat. Rev. Drug Discov. 6(11), 881-890 (2007).

14 Gleeson MP, Modi S, Bender A et al. The challenges involved in modeling toxicity data in silico: a review. Curr. Pham Des. 18(9), 1266-1291 (2012).

15 Blum LC, Reymond J-L. 970 million druglike small molecules for virtual screening in the chemical universe database GDB-13. J. Am. Chem. Soc. 131(25), 8732-8733 (2009).

16 Irwin JJ, Shoichet BK. ZINC - a free database of commercially available compounds for virtual screening. J. Chem. Inf. Comput. Sci. 45(1), 177-182 (2005).

17 The gold standard for chemical substance information. www.cas.org/content/chemical-substances

18 eMolecules: Linking researchers needs to supplier capabilities. www.emolecules.com

19 Bakken GA, Boehm M, Bell AS et al. Shaping a screening file for maximal lead discovery efficiency and effectiveness: elimination of molecular redundancy. J. Chem. Inf. Mod. 52, 2937-2949 (2012).

20 Baum R. Combinatorial chemistry. Chem. Eng. News Arch. 74(7), 28-73 (1996).

21 Baell JB. Broad coverage of commercially available lead-like screening space with fewer than 350,000 compounds. J. Chem. Inf. Mod. 53, 39-55 (2013).

- Describes important aspects of library construction and some key roles chemical vendors can play in enhancing the quality of screening libraries.

22 Walters WP, Namchuk M. A guide to drug discovery: designing screens: how to make your hits a hit. Nat. Rev. Drug Discov. 2(4), 259-266 (2003).

23 Lovering F, Bikker J, Humblet C. Escape from Flatland: increasing saturation as an approach to improving clinical success. J. Med. Chem. 52(21), 6752-6756 (2009). 
24 Walters WP. Going further than Lipinski's rule in drug design. Exp. Opin. Drug Discov. 7(2), 99-107 (2012).

25 Oprea TI, Bologa CG, Boyer $S$ et al. A crowdsourcing evaluation of the NIH chemical probes. Nat. Chem. Biol. 5(7), 441-447 (2009).

26 Lajiness MS, Maggiora GM, Shanmugasundaram V. Assessment of the consistency of medicinal chemists in reviewing sets of compounds. J. Med. Chem. 47(20), 4891-4896 (2004).

27 Kutchukian PS, Vasilyeva NY, Xu J et al. Inside the mind of a medicinal chemist: the role of human bias in compound prioritization during drug discovery. PLoS ONE 7(11), e48476 (2012).

28 Bologa CG, Oprea TI. Compound collection preparation for virtual screening. Methods Mol. Biol. 910, 125-143 (2012).

29 Accelrys: Pipeline Pilot. www.accelrys.com/products/pipeline-pilot/

30 Schrödinger: Small-Molecule Drug Discovery Suite. www.schrodinger.com/smdd/

31 Dewitt SH, Czarnik AW. Combinatorial organic synthesis using Parke-Davis's diversomer method. Acc. Chem. Res. 29(3), 114-122 (1996).

32 Booth RJ, Hodges JC. Polymer-supported quenching reagents for parallel purification. J. Am. Chem. Soc. 119(21), 4882-4886 (1997).

33 Baell JB, Holloway GA. New substructure filters for removal of pan assay interference compounds (PAINS) from screening libraries and for their exclusion in bioassays. J. Med. Chem. 53(7), 2719-2740 (2010).

-• Seminal manuscript first reporting pan-assay interference compounds substructures.

34 Erlanson DA. Introduction to fragment-based drug discovery. Top. Curr. Chem. 317, 1-32 (2012).

35 Albert JS, Blomberg N, Breeze AL et al. An integrated approach to fragment-based lead generation: philosophy, strategy and case studies from AstraZeneca's drug discovery programmes. Curr. Top. Med. Chem. 7(16), 1600-1629 (2007).

36 Ferenczy GG, Keseru GM. How are fragments optimized? A retrospective analysis of 145 fragment optimizations. J. Med. Chem. 56(6), 2478-2486 (2013).

37 Eitner K, Koch U. From fragment screening to potent binders: strategies for fragment-to-lead evolution. Mini Rev. Med. Chem. 9(8), 956-961 (2009).

38 Wildman SA. Approaches to virtual screening and screening library selection. Curr. Pharm. Des. 19(26), 4787-4796 (2013).

39 Scior T, Bender A, Tresadern G et al. Recognizing pitfalls in virtual screening: a critical review. J. Chem. Inf. Mod. 52(4), 867-881 (2012).

40 Bleicher KH, Boehm H-J, Mueller K, Alanine AI. A guide to drug discovery: hit and lead generation: beyond highthroughput screening. Nat. Rev. Drug Discov. 2(5), 369-378 (2003).

41 ChEMBL (02-09-2014).

www.ebi.ac.uk/chembl/
42 Bento AP, Gaulton A, Hersey A et al. The ChEMBL bioactivity database: an update. Nucleic Acids Res 42(D1), D1083-D1090 (2014).

43 The PubChem Project. www.pubchem.ncbi.nlm.nih.gov

44 Lagalwar S, Orr HT. Regulation of ataxin-1 phosphorylation and its impact on biology. Methods Mol. Biol. 1010, 201-209 (2013).

45 Setyawan J, Koide K, Diller TC et al. Inhibition of protein kinases by balanol: specificity within the serine/threonine protein kinase subfamily. Mol. Pharm. 56(2), 370-376 (1999).

46 Akamine P, Madhusudan, Brunton LL et al. Balanol analogues probe specificity determinants and the conformational malleability of the cyclic 3',5'-adenosine monophosphate-dependent protein kinase catalytic subunit. Biochemistry 43, 85-96 (2004).

47 Manning G, Whyte DB, Martinez R, Hunter T, Sudarsanam $S$. The protein kinase complement of the human genome. Science 298(5600), 1912-1934 (2002).

48 Yan SF, Asatryan H, Li J, Zhou Y. Novel statistical approach for primary high-throughput screening hit selection. J. Chem. Inf. Mod. 45(6), 1784-1790 (2005).

49 Lessene G, Czabotar PE, Sleebs BE et al. Structure-guided design of a selective BCL-X $\mathrm{L}_{\mathrm{L}}$ inhibitor. Nat. Chem. Biol. 9, 390-397 (2013).

50 Morwick T, Berry A, Brickwood J et al. Evolution of the thienopyridine class of inhibitors of IkB kinase-beta: part I: hit-to-lead strategies. J. Med. Chem. 49, 2898-2908 (2006).

51 Qian K, Wang L, Cywin CL et al. Hit to lead account of the discovery of a new class of inhibitors of Pim kinases and crystallographic studies revealing an unusual kinase binding mode. J. Med. Chem. 52, 1814-1827 (2009).

52 Vidler LR, Filippakopoulos P, Fedorov O et al. Discovery of novel small-molecule inhibitors of BRD4 using structurebased virtual screening. J. Med. Chem. 56, 8073-8088 (2013).

53 Keseru GM, Makara GM. Hit discovery and hit-to-lead approaches. Drug Discov. Today 11, 741-748 (2006).

54 Macarron R, Banks MN, Bojanic D et al. Impact of highthroughput screening in biomedical research. Nat. Rev. Drug Discov. 10, 188-195 (2011).

55 Schonbrunn E, Betzi S, Alam R et al. Development of highly potent and selective diaminothiazole inhibitors of cyclin-dependent kinases. J. Med. Chem. 56(10), 3768-3782 (2013).

56 Hopkins A, Groom C. Ligand efficiency: a useful metric for lead selection. Drug Discov. Today 9(10), 430-431 (2004).

57 Harper G, Pickett SD. Methods for mining HTS data. Drug Discov. Today 11(15-16), 694-699 (2006).

58 Malo N, Hanley JA, Cerquozzi S, Pelletier J, Nadon R. Statistical practice in high-throughput screening data analysis. Nat. Biotech. 24(2), 167-175 (2006).

59 Zhang J, Chung T, Oldenburg K. A simple statistical parameter for use in evaluation and validation of high throughput screening assays. J. Biomol. Screen. 4(2), 67-73 (1999). 
60 Gubler H. Methods for statistical analysis, quality assurance and management of primary high-throughput screening data. In: High-Throughput Screening in Drug Discovery. Hüser J (Ed.). Wiley-VCH Verlag GmbH \& Co. KGaA, Weinheim, Germany (2006).

61 Malo N, Hanley JA, Carlile G et al. Experimental design and statistical methods for improved hit detection in highthroughput screening. J. Biomol. Screen. 15(8), 990-1000 (2010).

- Highlights several benefits of integrating statistical corrections into the HTS process.

62 Makarenkov V, Kevorkov D, Zentilli P, Gagarin A, Malo N, Nadon R. HTS-Corrector: software for the statistical analysis and correction of experimental high-throughput screening data. Bioinformatics 22(11), 1408-1409 (2006).

63 Gunter B, Brideau C, Pikounis B, Liaw A. Statistical and graphical methods for quality control determination of high-throughput screening data. J. Biomol. Screen. 8(6), 624-633 (2003).

64 Inglese J, Auld DS, Jadhav A et al. Quantitative highthroughput screening: A titration-based approach that efficiently identifies biological activities in large chemical libraries. Proc. Natl Acad. Sci. USA 103(31), 11473-11478 (2006).

65 Shapiro AB, Walkup GK, Keating TA. Correction for interference by test samples in high-throughput assays. J. Biomol. Screen. 14(8), 1008-1016 (2009).

66 Brideau C, Gunter B, Pikounis B, Liaw A. Improved statistical methods for hit selection in high-throughput screening. J. Biomol. Screen. 8(6), 634-647 (2003).

67 Baell JB. Observations on screening-based research and some concerning trends in the literature. Future Med. Chem. 2(10), 1529-1546 (2010).

68 Saubern S, Guha R, Baell JB. KNIME workflow to assess PAINS filters in SMARTS Format. Comparison of RDKit and Indigo cheminformatics libraries. Mol. Inform. 30 (10), 847-850 (2011).

69 Baell JB, Ferrins L, Falk H, Nikolakopoulos G. PAINS: Relevance to tool compound discovery and fragment-based screening. Aust. J. Chem. 66(12), 1483-1494 (2013).

70 Dahlin JL, Sinville R, Solberg J et al. A cell-free fluorometric high-throughput screen for inhibitors of Rtt109-catalyzed histone acetylation. PLoS ONE 8(11), e78877 (2013).

71 Bancos I, Bida JP, Tian D et al. High-throughput screening for growth inhibitors using a yeast model of familial paraganglioma. PLoS ONE 8(2), e56827 (2013).

72 Bruns RF, Watson IA. Rules for identifying potentially reactive or promiscuous compounds. J. Med. Chem. 55(22), 9763-9772 (2012).

73 Tomaši T, Maši LP. Rhodanine as a scaffold in drug discovery: a critical review of its biological activities and mechanisms of target modulation. Exp. Opin. Drug Discov. 7(7), 549-60 (2012).

74 Wang Y, Cesena TI, Ohnishi Y et al. Small molecule screening identifies regulators of the transcription factor $\triangle$ FosB. ACS Chem. Neurosci. 3, 546-556 (2012).
75 Metz JT, Huth JR, Hajduk PJ. Enhancement of chemical rules for predicting compound reactivity towards protein thiol groups. J. Comput. Aid. Mol. Des. 21(1-3), 139-144 (2007).

76 Rishton G. Reactive compounds and in vitro false positives in HTS. Drug Discov. Today 2, 382-384 (1997).

77 Fliege R, Metzler M. Electrophilic properties of patulin. $N$-acetylcysteine and glutathione adducts. Chem. Res. Toxicol. 13(5), 373-381 (2000).

78 Huth JR, Song D, Mendoza RR et al. Toxicological evaluation of thiol-reactive compounds identified using a la assay to detect reactive molecules by nuclear magnetic resonance. Chem. Res. Toxicol. 20(12), 1752-1759 (2007).

79 Mirkovi B, Sosi I, Gobec S, Kos J. Redox-based inactivation of cysteine cathepsins by compounds containing the 4-aminophenol moiety. PLoS ONE 6(11), e27197 (2011).

80 Johnston PA. Redox cycling compounds generate $\mathrm{H}_{2} \mathrm{O}_{2}$ in HTS buffers containing strong reducing reagents-real hits or promiscuous artifacts? Curr. Opin. Chem. Biol. 15(1), 174-182 (2011).

81 Johnston PA, Soares KM, Shinde SN et al. Development of a 384-well colorimetric assay to quantify hydrogen peroxide generated by the redox cycling of compounds in the presence of reducing agents. Assay Drug Dev. Technol. 6(4), 505-518 (2008).

82 Coan KED, Maltby DA, Burlingame AL, Shoichet BK. Promiscuous aggregate-based inhibitors promote enzyme unfolding. J. Med. Chem. 52(7), 2067-2075 (2009).

83 Coan KED, Shoichet BK. Stoichiometry and physical chemistry of promiscuous aggregate-based inhibitors. J. Am. Chem. Soc. 130(29), 9606-9612 (2008).

84 Feng BY, Simeonov A, Jadhav A et al. A high-throughput screen for aggregation-based inhibition in a large compound library. J. Med. Chem. 50 (10), 2385-2390 (2007).

85 Jadhav A, Ferreira RS, Klumpp C et al. Quantitative analyses of aggregation, autofluorescence, and reactivity artifacts in a screen for inhibitors of a thiol protease. J. Med. Chem. 53(1), 37-51 (2010).

86 Seidler J, Mcgovern SL, Doman TN, Shoichet BK. Identification and prediction of promiscuous aggregating inhibitors among known drugs. J. Med. Chem. 46(21), 4477-4486 (2003).

87 Mcgovern S, Helfand B, Feng B. A specific mechanism of nonspecific inhibition. J. Med. Chem. 46, 4265-4272 (2003).

88 Mcgovern SL, Caselli E, Grigorieff N, Shoichet BK. A common mechanism underlying promiscuous inhibitors from virtual and high-throughput screening. J. Med. Chem. 45(8), 1712-1722 (2002).

89 Ryan AJ, Gray NM, Lowe PN, Chung C-W. Effect of detergent on 'promiscuous' inhibitors. J. Med. Chem. 46(16), 3448-3451 (2003).

90 Laplante SR, Carson R, Gillard J et al. Compound aggregation in drug discovery: implementing a practical NMR assay for medicinal chemists. J. Med. Chem. 56(12), 5142-5150 (2013). 
91 Huth JR, Mendoza R, Olejniczak ET et al. ALARM NMR: a rapid and robust experimental method to detect reactive false positives in biochemical screens. J. Am. Chem. Soc. 127(1), 217-224 (2005).

- Describes a protein-based NMR method to probe for protein-reactive compounds.

92 Mccallum MM, Nandhikonda P, Temmer JJ et al. Highthroughput identification of promiscuous inhibitors from screening libraries with the use of a thiol-containing fluorescent probe. J. Biomol. Screen. 18(6), 705-713 (2013).

93 Epps DE, Taylor BM. A competitive fluorescence assay to measure the reactivity of compounds. Anal. Biochem. 295(1), 101-106 (2001).

94 Böhme A, Thaens D, Paschke A, Schüürmann G. Kinetic glutathione chemoassay to quantify thiol reactivity of organic electrophiles - application to alpha, beta-unsaturated ketones, acrylates, and propiolates. Chem. Res. Toxicol. 22(4), 742-750 (2009).

95 Avonto C, Taglialatela-Scafati O, Pollastro F et al. An NMR spectroscopic method to identify and classify thiol-trapping agents: revival of Michael acceptors for drug discovery? Ange Chem. 50(2), 467-471 (2011).

96 Schebb NH, Faber H, Maul R et al. Analysis of glutathione adducts of patulin by means of liquid chromatography (HPLC) with biochemical detection (BCD) and electrospray ionization tandem mass spectrometry (ESI-MS/MS). Anal. Bioanal. Chem. 394(5), 1361-1373 (2009).

97 Wei C, Chupak L, Philip T, Johnson B, Gentles R, Drexler D. Screening and characterization of reactive compounds with in vitro peptide-trapping and liquid chromatography/ high-resolution accurate mass spectrometry. J. Biomol. Screen. 19(2), 297-307 (2014).

98 Falk H, Connor T, Yang H et al. An efficient highthroughput screening method for MYST family acetyltransferases, a new class of epigenetic drug targets. J. Biomol. Screen. 16(10), 1196-1205 (2011).

99 Schorpp K, Rothenaigner I, Salmina E et al. Identification of small-molecule frequent hitters from AlphaScreen high-throughput screens. J. Biomol. Screen. 19(5), 715-726 (2013).

100 Simeonov A, Yasgar A, Klumpp C et al. Evaluation of microparallel liquid chromatography as a method for HTS-coupled actives verification. Assay Drug Dev. Technol. 5(6), 815-824 (2007).

101 Thorne N, Auld DS, Inglese J. Apparent activity in highthroughput screening: origins of compound-dependent assay interference. Curr. Opin. Chem. Biol. 14(3), 315-324 (2010).

102 Auld DS, Southall NT, Jadhav A et al. Characterization of chemical libraries for luciferase inhibitory activity. J. Med. Chem. 51(8), 2372-2386 (2008).

103 Heitman LH, Veldhoven JPDV, Zweemer AM, Ye K, Brussee J, Ijzerman AP. False positives in a reporter gene assay: Identification and synthesis of substituted $N$-pyridin-2ylbenzamides as competitive inhibitors of firefly luciferase. J. Med. Chem. 51(15), 4724-4729 (2008).

104 Auld DS, Thorne N, Maguire WF, Inglese J. Mechanism of PTC124 activity in cell-based luciferase assays of nonsense codon suppression. Proc. Natl Acad. Sci. USA 106(9), 3585-3590 (2009).

105 Auld DS, Lovell S, Thorne N et al. Molecular basis for the high-affinity binding and stabilization of firefly luciferase by PTC124. Proc. Natl Acad. Sci. USA 107(11), 4878-4883 (2010).

-• Part of a well-designed series of experiments demonstrating compound-reporter assay interference.

106 Simeonov A, Jadhav A, Thomas CJ et al. Fluorescence spectroscopic profiling of compound libraries. J. Med. Chem. 51(8), 2363-2371 (2008).

107 Turek-Etienne TC, Small EC, Soh SC et al. Evaluation of fluorescent compound interference in 4 fluorescence polarization assays: 2 kinases, 1 protease, and 1 phosphatase. J. Biomol. Screen. 8(2), 176-184 (2003).

108 Beasley JR, Dunn DA, Walker TL, Parlato SM, Lehrach JM, Auld DS. Evaluation of compound interference in immobilized metal ion affinity-based fluorescence polarization detection with a four million member compound collection. Assay Drug Dev. Technol. 1(3), 455-459 (2003).

109 Imbert P-E, Unterreiner V, Siebert D, Gubler H, Parker C, Gabriel D. Recommendations for the reduction of compound artifacts in time-resolved fluorescence resonance energy transfer assays. Assay Drug Dev. Technol. 5(3), 363-372 (2007).

110 Pai JJ, Kirkup MP, Frank EA, Pachter JA, Bryant RW. Compounds capable of generating singlet oxygen represent a source of artifactual data in scintillation proximity assays measuring phosphopeptide binding to $\mathrm{SH} 2$ domains. Anal. Biochem. 270(1), 33-40 (1999).

111 Canny SA, Cruz Y, Southern MR, Griffin PR. PubChem promiscuity: a web resource for gathering compound promiscuity data from PubChem. Bioinformatics 28(1), 140-141 (2009).

112 Han L, Wang Y, Bryant SH. A survey of across-target bioactivity results of small molecules in PubChem. Bioinformatics 25(17), 2251-2255 (2009).

$113 \mathrm{Hu} \mathrm{Y,} \mathrm{Bajorath} \mathrm{J.} \mathrm{What} \mathrm{is} \mathrm{the} \mathrm{likelihood} \mathrm{of} \mathrm{an} \mathrm{active}$ compound to be promiscuous? Systematic assessment of compound promiscuity on the basis of PubChem confirmatory bioassay data. AAPS J. 15(3), 808-815 (2013).

114 Prinz H. Simplification traps. J. Chem. Biol. 5(1), 1-4 (2012).

115 Prinz H. Hill coefficients, dose-response curves and allosteric mechanisms. J. Chem. Biol. 3(1), 37-44 (2010).

116 Shoichet BK. Interpreting steep dose-response curves in early inhibitor discovery. J. Med. Chem. 49(25), 7274-7277 (2006).

117 Hattori K, Wakabayashi H, Tamaki K. Predicting key example compounds in competitors' patent applications using structural information alone. J. Chem. Inf. Model. 48(1), 135-142 (2008).

118 Muchmore SW, Edmunds JJ, Stewart KD, Hajduk PJ. Cheminformatic tools for medicinal chemists. J. Med. Chem. 53(13), 4830-4841 (2010).

119 Oprea TI, Bologa CG, Edwards BS, Prossnitz ER, Sklar LA. Post-high-throughput screening analysis: an empirical 
compound prioritization scheme. J. Biomol. Screen. 10(5), 419-426 (2005).

120 Duffy BC, Zhu L, Decornez H, Kitchen DB. Early phase drug discovery: cheminformatics and computational techniques in identifying lead series. Bioorg. Med. Chem. 20(18), 5324-5342 (2012).

- A well-written review of the hit and lead identification stages in early drug discovery, with excellent descriptions of how cheminformatics can enhance this process.

121 Wawer M, Bajorath J. Extraction of structure-activity relationship information from high-throughput screening data. Curr. Med. Chem. 16(31), 4049-4057 (2009).

122 Cox PB, Gregg RJ, Vasudevan A. Abbott Physicochemical Tiering (APT) - a unified approach to HTS triage. Bioorg. Med. Chem. 20(14), 4564-4573 (2012).

123 Lipinski CA, Lombardo F, Dominy BW, Feeney PJ. Experimental and computational approaches to estimate solubility and permeability in drug discovery and development settings. Adv. Drug Deliv. Rev. 23(1-3), 3-25 (1997).

124 Veber DF, Johnson SR, Cheng H-Y, Smith BR, Ward KW, Kopple KD. Molecular properties that influence the oral bioavailability of drug candidates. J. Med. Chem. 45(12), 2615-2623 (2002).

125 Baell J, Congreve M, Leeson P, Abad-Zapatero C. Ask the experts: past, present and future of the rule of five. Future Med. Chem. 5(7), 745-752 (2013).

126 Hopkins AL, Keserue GM, Leeson PD, Rees DC, Reynolds $\mathrm{CH}$. The role of ligand efficiency metrics in drug discovery. Nat. Rev. Drug Discov. 13(2), 105-121 (2014).

127 Freeman-Cook KD, Hoffman RL, Johnson TW. Lipophilic efficiency: the most important efficiency metric in medicinal chemistry. Future Med. Chem. 5(2), 113-115 (2013).

128 Abad-Zapatero C. Ligand efficiency indices for effective drug discovery. Exp. Opin. Drug Discov. 2(4), 469-488 (2007).

129 Abad-Zapatero C, Blasi D. Ligand efficiency indices (LEIs): more than a simple efficiency yardstick. Mol. Inform. 30(2-3), 122-132 (2011)

130 Abad-Zapatero C, Metz JT. Ligand efficiency indices as guideposts for drug discovery. Drug Discov. Today 10(7), 464-469 (2005).

131 Šink R, Gobec S, Pecar S. False positives in the early stages of drug discovery. Curr. Med. Chem. 17, 4231-4255 (2010).

132 Cumming JG, Davis AM, Muresan S, Haeberlein M, Chen H. Chemical predictive modelling to improve compound quality. Nat. Rev. Drug Discov. 12(12), 948-962 (2013).

133 Neuwald A, Landsman D. GCN5-related histone $N$ acetyltransferases belong to a diverse superfamily that includes the yeast SPT10 protein. Trends Biochem. Sci. 22, 154-155 (1997).

134 Wager TT, Hou X, Verhoest PR, Villalobos A. Moving beyond rules: the development of a Central Nervous System Multiparameter Optimization (CNS MPO) approach to enable alignment of druglike properties. ACS Chem. Neurosci. 1(6), 435-449 (2010).

135 Zhu T, Cao S, Su P-C et al. Hit identification and optimization in virtual screening: practical recommendations based on a critical literature analysis. J. Med. Chem. 56(17), 6560-6572 (2013).

136 Tanrikulu Y, Kruger B, Proschak E. The holistic integration of virtual screening in drug discovery. Drug Discov. Today 18(7-8), 358-364 (2013).

137 Lagorce D, Maupetit J, Baell J et al. The FAF-Drugs2 server: a multistep engine to prepare electronic chemical compound collections. Bioinformatics 27(14), 2018-2020 (2011).

138 Park J-G, Kahn JN, Tumer NE, Pang Y-P. Chemical structure of Retro-2, a compound that protects cells against ribosome-inactivating proteins. Sci. Rep. 2, 631 (2012).

139 Kozikowski BA, Burt TM, Tirey DA et al. The effect of room-temperature storage on the stability of compounds in DMSO. J. Biomol. Screen. 8(2), 205-209 (2003).

140 Kozikowski BA, Burt TM, Tirey DA et al. The effect of freeze/thaw cycles on the stability of compounds in DMSO. J. Biomol. Screen. 8(2), 210-215 (2003).

141 Blaxill Z, Holland-Crimmin S, Lifely R. Stability through the ages: the GSK experience. J. Biomol. Screen. 14(5), 547-556 (2009).

142 Ellson R, Stearns R, Mutz M et al. In situ DMSO hydration measurements of HTS compound libraries. Comb. Chem. High Throughput Screen. 8(6), 489-498 (2005).

143 Engeloch C, Schopfer U, Muckenschnabel I et al. Stability of screening compounds in wet DMSO. J. Biomol. Screen. 13(10), 999-1006 (2008).

144 Matson SL, Chatterjee M, Stock DA et al. Best practices in compound management for preserving compound integrity and accurately providing samples for assays. J. Biomol. Screen. 14(5), 476-484 (2009).

145 Kenseth JR, Coldiron SJ. High-throughput characterization and quality control of small-molecule combinatorial libraries. Curr. Opin. Chem. Biol. 8(4), 418-423 (2004).

146 Wipf P, Arnold D, Carter K et al. A case study from the chemistry core of the Pittsburgh Molecular Library Screening Center: the Polo-like kinase polo-box domain (Plk1-PBD). Curr. Topics Med. Chem. 9(13), 1194-1205 (2009).

147 Hermann JC, Chen Y, Wartchow C et al. Metal impurities cause false positives in high-throughput screening campaigns. ACS Med. Chem. Lett. 4(2), 197-200 (2013).

148 Tarzia G, Antonietti F, Duranti A et al. Identification of a bioactive impurity in a commercial sample of 6-methyl-2p-tolylaminobenzo[d][1,3] oxazin-4-one (URB754). Ann. Chim. 97(9), 887-894 (2007).

- An important case study demonstrating the importance of analytical chemistry and chemical resynthesis in post-HTS triage.

149 Guntupalli S, Ray UK, Murali N et al. Identification, isolation and characterization of process related impurities in ezetimibe. J. Pharm. Biomed. Anal. 88, 385-390 (2014).

150 Liu M, Wang J, Wu X et al. HPLC method development, validation and impurity characterization for an antitumor Hsp90 inhibitor-PU-H71 (NSC 750424). J. Pharm. Biomed. Anal. 89, 34-41 (2014).

151 Verbeken M, Wynendaele E, Lefebvre RA, Goossens E, Spiegeleer BD. The influence of peptide impurity profiles on 
functional tissue-organ bath response: the 11-mer peptide INSL6[151-161] case. Anal. Biochem. 421(2), 547-555 (2012).

152 Dotterer SK, Forbes RA, Hammill CL. Impact of metalinduced degradation on the determination of pharmaceutical compound purity and a strategy for mitigation. J. Pharm. Biomed. Anal. 54(5), 987-994 (2011).

153 Jaki BU, Frazblau SG, Chadwick LR et al. Purity-activity relationships of natural products: the case of anti-TB active ursolic acid. J. Nat. Prod. 71, 1742-1748 (2008).

154 Ekins S, Freundlich JS, Hobrath JV, Lucile White E, Reynolds RC. Combining computational methods for hit to lead optimization in Mycobacterium tuberculosis drug discovery. Pharm. Res. 31(2), 414-435 (2014).

155 Zhang L, Wang X, Feng J, Jia Y, Xu F, Xu W. Discovery of novel vascular endothelial growth factor receptor 2 inhibitors: a virtual screening approach. Chem. Biol. Drug Des. 80(6), 893-901 (2012).

156 Zhang X-F, Tan M, Chhabra M, Dai Y-C, Meller J, Jiang X. Inhibition of histo-blood group antigen binding as a novel strategy to block Norovirus infections. PLoS ONE 8(7), e69379 (2013).

157 Zhu P, Peng H, Ni N, Wang B, Li M. Novel AI-2 quorum sensing inhibitors in Vibrio harveyi identified through structure-based virtual screening. Bioorg. Med. Chem. Lett. 22(20), 6413-6417 (2012).

158 Zhuang C, Narayanapillai S, Zhang W, Sham YY, Xing C. Rapid identification of Keap1-Nrf2 small-molecule inhibitors through structure-based virtual screening and hit-based substructure search. J. Med. Chem. 57(3), 1121-1126 (2014).

159 Liang Z, Ding X, Ai J et al. Discovering potent inhibitors against c-Met kinase: molecular design, organic synthesis and bioassay. Org. Biomol. Chem. 10(2), 421-430 (2012).

160 Mukherjee P, Desai P, Ross L, White EL, Avery MA. Structure-based virtual screening against SARS-3CLpro to identify novel non-peptidic hits. Bioorg. Med. Chem. 16(7), 4138-4149 (2008)

161 Chen L, Chen S, Gui C, Shen J, Shen X, Jiang H. Discovering severe acute respiratory syndrome coronavirus 3CL protease inhibitors: virtual screening, surface plasmon resonance, and fluorescence resonance energy transfer assays. J. Biomol. Screen. 11(8), 915-921 (2006).

162 Han C, Zhang J, Chen L, Chen K, Shen X, Jiang H. Discovery of Helicobacter pylori shikimate kinase inhibitors: bioassay and molecular modeling. Bioorg. Med Chem. 15(2), 656-662 (2007).

163 Schepetkin IA, Khlebnikov AI, Kirpotina LN, Quinn MT. Novel small-molecule inhibitors of anthrax lethal factor identified by high-throughput Screening. J. Med. Chem. 49(17), 5232-5244 (2006).
164 Xie Y, Liu Y, Gong G et al. Discovery of a novel submicromolar inhibitor of the lymphoid specific tyrosine phosphatase. Bioorg. Med. Chem. Lett. 18(9), 2840-2844 (2008).

165 Xu F, Zhang L, Jia Y et al. Discovery of 4-amino-2-(thio) phenol derivatives as novel protein kinase and angiogenesis inhibitors for the treatment of cancer: synthesis and biological evaluation. Eur. J. Med. Chem. 69, 191-200 (2013).

166 Qin J, Xie P, Ventocilla C et al. Identification of a novel family of BRAFV600E inhibitors. J. Med. Chem. 55, 5220-5230 (2012).

167 Ge Y, Kazi A, Marsilio F et al. Discovery and synthesis of hydronaphthoquinones as novel proteasome inhibitors. J. Med. Chem. 55(5), 1978-1998 (2012).

168 Soares KM, Blackmon N, Shun TY et al. Profiling the NIH Small Molecule Repository for compounds that generate $\mathrm{H}_{2} \mathrm{O}_{2}$ by redox cycling in reducing environments. Assay Drug Dev. Technol. 8(2), 152-174 (2010).

169 Kilgore JA, Du X, Melito L et al. Identification of DNMT1 selective antagonists using a novel scintillation proximity assay. J. Biol. Chem. 288(27), 19673-19684 (2013).

170 Nagarajan S, Skoufias DA, Kozielski F, Pae AN. Receptor-ligand interaction-based virtual screening for novel Eg5/kinesin spindle protein inhibitors. J. Med. Chem. 55(6), 2561-2573 (2012).

171 Park I-H, Li C. Characterization of molecular recognition of STAT3 SH2 domain inhibitors through molecular simulation. J. Mol. Recognit. 24, 254-265 (2011).

172 Xu X, Kasembeli MM, Jiang X, Tweardy BJ, Tweardy DJ. Chemical probes that competitively and selectively inhibit Stat3 activation. PLoS ONE 4(3), e4783 (2009).

173 Gilson MK, Georg G, Wang S. Digital chemistry in the Journal of Medicinal Chemistry. J. Med. Chem. 57(4), 1137-1137 (2014).

174 Kalgutkar AS, Dalvie DK. Drug discovery for a new generation of covalent drugs. Exp. Opin. Drug Discov. 7(7), 561-581 (2012).

175 Vince R. A brief history of the development of Ziagen. Chemtracts-Org. Chem. 21(4), 127-134 (2008).

176 Dosa PI, Georg GI, Gurvich VJ, Hawkinson JE, Walters MA, Wong HL. From HTS to Phase I: The Institute for Therapeutics Discovery and Development at the University of Minnesota. Comb. Chem. High Throughput Screen. 17(3), 231-240 (2014).

177 CAS www.cas.org 